To be submitted to A.J.

\title{
The Vela Cloud: A Giant HI Anomaly in the NGC 3256 Group
}

\author{
Jayanne English \\ University of Manitoba \\ B. Koribalski \\ Australia Telescope National Facility, CSIRO \\ J. Bland-Hawthorn \\ Anglo-Australian Observatory \\ K. C. Freeman \\ Research School of Astronomy and Astrophysics, The Australian National University \\ Claudia F. McCain \\ Research School of Astronomy and Astrophysics, The Australian National University
}

\begin{abstract}
We present Australia Telescope Compact Array (ATCA) observations of a galaxy-sized intergalactic HI cloud ("the Vela Cloud") in the NGC 3256 galaxy group. The group contains the prominent merging galaxy NGC 3256, which is surrounded by a number of HI fragments, the tidally disturbed galaxy NGC 3263, and several other peculiar galaxies. The Vela Cloud, with an HI mass of 3-5 $\times 10^{9} \mathcal{M}_{\odot}$, resides southeast of NGC 3256 and west of NGC 3263, within an area of $9^{\prime} \times 16^{\prime}(100 \mathrm{kpc} \times 175 \mathrm{kpc}$ for an adopted distance of $38 \mathrm{Mpc})$. In our ATCA data the Vela Cloud appears as 3 diffuse components and contains 4 density enhancements. The Vela Cloud's properties, together with its group environment, suggest that it has a tidal origin. Each density enhancement contains $\sim 10^{8} \mathcal{M}_{\odot}$ of HI gas which is sufficient material for the formation of globular cluster progenitors. However, if we represent the enhancements as Bonnor-Ebert spheres, then the pressure of the surrounding HI would need to increase by at least a factor of 9 in order to cause the collapse of an enhancement. Thus we do not expect them to form massive bound stellar systems like super star clusters or dwarf galaxies.
\end{abstract}


Since the HI density enhancements have some properties in common with compact High Velocity Clouds, we explore whether they may evolve to be identified with these starless clouds instead.

Subject headings: galaxies: individual (NGC3256, NGC3263) — galaxies: evolution - galaxies: interactions - galaxies: intergalactic medium

\section{Introduction}

\subsection{Intergalactic clouds}

The detection of intergalactic clouds, such as the one studied in this paper, can illuminate the progress of galaxy evolution. For example, one expects to find primordial HI building blocks of hierarchical galaxy construction unless galaxy formation was extremely efficient. However these are rarer than predicted by the current cosmological theories which build galaxies via the hierarchical merging of proto-galactic clouds embedded in dark-matter halos (e.g. Klypin et al. 1999). Studies of groups of galaxies reveal very few metal-poor and star-poor clouds (e.g. Zwaan 2001 and papers referenced therein; Pisano et al. 2004). Other HI detections include bona fide dwarf galaxies (e.g. Côté et al. 2000); HI envelopes enclosing superwinds (e.g. Ott et al. 2005); and outflows from disks due to supernovae or buoyantly rising bubbles (e.g. Irwin \& Chaves (2003) and papers mentioned therein) or plumes potentially due to ram pressure (e.g. Oosterloo \& van Gorkom 2005). A strongly accepted explanation for many HI clouds is that they are tidal extensions from galaxies and/or tidal dwarf galaxies (e.g. Duc et al. 2004; Hibbard et al. 2001a; Hibbard et al. 2001b; Koribalski et al. 2003; Koribalski \& Dickey 2004; Koribalski 2004). Tidal origins are also proposed for HI companions to HII galaxies (Taylor et al. (1993, 1995, 1996), the compact HI companion to FCC 35 (Putman et al. 1998), and the extended companion to NGC 2442 called HIPASS J0731-69 (Ryder et al. 2001). Indeed the Leo Ring (Schneider et al. 1983) is associated with a group of galaxies and is likely to have a tidal origin (Bekki et al. 2005b).

A similar debate also exists about the origins of the Galactic High Velocity Clouds (HVCs). Since stars in HVC have not yet been detected, their distances from the Milky Way are difficult to determine. Recent studies indicate that they are within tens of kpc (e.g. Kalberla \& Haud 2006 and references mentioned within; Wakker et al. 2008). HVCs could be nearby tidal debris similar to fragments of the Magellanic Stream (e.g. Westmeier \& Koribalski 2008, Putman et al. 2003a, Putman et al. 2003b) or material cooling and infalling from a primordial halo (e.g. Peek et al. 2006 and references mentioned therein). Another alternative is that they are material ejected from the disk of our Galaxy to a height of a few kpc which 
subsequently rains back down onto the plane as a Galactic fountain (e.g. Norman \& Ikeuchi 1989; Ganguly et al. 2005).

In order to clarify the origins of HVCs, there are numerous studies of extra-planar HI associated with individual galaxies. Since the detected HI clouds are in the vicinity of the host galaxies the origins of these clouds are interpreted as either a galactic fountain (e.g. Fraternali \& Binney 2008) or tidal debris (e.g. Westmeier et al. 2007) or possibly a condensation from a primordial halo (e.g. Rand \& Benjamin 2008).

A survey for extra-galactic HVCs in groups of galaxies by Pisano et al. (e.g. 2004, 2007, and papers mentioned therein) have detected optical counterparts to HI clouds, suggesting they are dwarf galaxies. Also the proximity of the dwarfs to individual galaxies within each group seems to argue against in-fall from outside a group's radius, excluding a cosmological origin. The on-going merger NGC 3256 also displays HI fragments in its vicinity (English et al. 2003). Since they appear to be starless, they may be analogous to HVCs.

\subsection{The Vela Cloud in the NGC 3256 Group}

The NGC 3256 Group and NGC 3263 Group are difficult to distinguish from each other spatially and the systemic velocities of their eponymous galaxies differ by less than 200 $\mathrm{km} \mathrm{s}^{-1}$. Additionally two different group-finding algorithms applied to the same dataset do not find the same galaxies in each group (Fouque et al. 1992; Garcia 1993). Many researchers therefore consider at least 15 galaxies to be members of a single large group spread over roughly a few degrees (eg. Lípari et al. 2000; Mould et al. 1991). We adopt this latter perspective for the remainder of the paper and refer to the association of galaxies as the NGC 3256 Group.

The most spectacular HI feature in the NGC 3256 Group is a galaxy-sized intergalactic HI cloud (English 1994), which we will refer to as "the Vela Cloud". The structure, as projected on the sky, is not clearly associated with an individual galaxy but appears to be part of the group. A tidal origin for the cloud is suggested by the fact that most of the galaxies in this region are tidally disturbed. This fact can be seen from those members of the group that are present in the field of view of our Australia Telescope Compact Array (ATCA) HI data (Fig. 11). The cloud's potential evolution is also interesting, and this paper explores whether parts of the cloud could be identified with HVCs at some point in their evolution.

The Vela Cloud was initially detected in an ATCA pointing towards NGC 3256 (English 1994; English et al. 2003) and further studied with 3 pointings of the Parkes Telescope 
English 1994). In this paper we present additional ATCA data with a pointing centre towards NGC 3263, which confirms the Vela Cloud's existence (Fig. 11). The Vela Cloud appears to be a cohesive structure, with 3 distinct diffuse gas sub-components, which we have labelled A, B, and C; see the schematic in Fig. 2, Four HI density enhancements are seen and an aim of this paper is to consider whether the internal pressure within these enhancements satisfies the conditions for initiating star formation.

Our radio and optical (broad band and Fabry-Perot) observations and reductions are described in $\S$ 2. $\S 3$ only describes measurement methods and the uncertainties associated with these methods. The actual measurements of the cloud's observed characteristics are detailed in section $\S$ 4. In $\S 5$ we describe derived characteristics (such as the amount of HI mass in emission), explore the star-forming potential in the Vela Cloud (representing the enhancements as Bonnor-Ebert spheres), and consider the ultraviolet radiation field of NGC 3263 in relation to the Vela Cloud. Then in $\S[$ the cloud's characteristics are contrasted with those of tidal debris, and other apparently isolated clouds, including HVCs. In $\S$ 7, we discuss the likely role of the group's tidal field on the origin of the Vela Cloud and speculate on the cloud's fate, asking whether its enhancements may evolve into HVCs. $\S 8$ summarizes our study of this rare galaxy-sized intergalactic cloud.

\section{Observations}

\subsection{Radio Observations and Reductions.}

21-cm line data were acquired using the ATCA in 1995 and 1996. The target centre was right ascension $\alpha=10^{\mathrm{h}} 29^{\mathrm{m}} 04^{\mathrm{s}}$ and declination $\delta=-44^{\circ} 04^{\prime} 12^{\prime \prime}(\mathrm{J} 2000)$, close to NGC 3263 . At $1406 \mathrm{MHz}$, with a bandwidth of $8 \mathrm{MHz}$, the observations spanned that galaxy's systemic and rotation velocities and included several other galaxies in the NGC 3256 Group. Array configurations $375,750 \mathrm{~A}, 1.5 \mathrm{~B}, 6 \mathrm{C}$ were used to acquire $12.5,10.2,11.6,10.7$ hours, respectively. The longest baseline corresponds to a spatial resolution of 7 arcsec while the primary beam is 33 arcmin. These 4 UV datasets were flagged and calibrated, using AIPS and the calibrators PKS 1934-638 (primary) and PKS 0823-500 (secondary), in the usual way. The continuum emission was fit using the line-free emission on either side of the HI signal and subtracted in the UV plane. After converting their frequency axes to optically defined heliocentric velocities, the datasets from the 4 array-configurations were combined.

For the subsequent Fourier transformation, deconvolution, and analysis we used the MIRIAD software package. We base our data analysis on a cube with the parameters listed in Table 1 and on subcubes which span smaller velocity ranges (see $\S$ 3). The characteristics 
of the cubes used to produce Fig. 1 and Fig. 3 are listed in those figure captions.

Although radio continuum observations were also made at $1380 \mathrm{MHz}$, the intergalactic cloud was not detected.

Single-dish telescope data were acquired on Dec. 19 and 20, 1993 using the single-feed 20-cm receiver at the 64-m Parkes Telescope, a bandwidth of $32 \mathrm{MHz}$, and 2 polarizations of 1024 channels each. The effective channel width is $8.2 \mathrm{~km} \mathrm{~s}^{-1}$. Observations were acquired for 3 different target positions on the sky. For each position 2 scans were acquired on-target along with 2 reference scans off-target. Each scan was 10 minutes. The off-target scans were subtracted from the on-target data and also used for normalization of the spectra; the r.m.s. is $0.013 \mathrm{Jy}$ per channel. We present one of the target positions $\left(\alpha=10^{\mathrm{h}} 28^{\mathrm{m}} 27^{\mathrm{s}} .7\right.$, $\left.\delta=-44^{\circ} 09^{\prime} 25^{\prime \prime}(\mathrm{J} 2000)\right)$ in this paper. While this pointing is west of NGC 3263 , the $15^{\prime}$ beam width encompasses some emission from this galaxy. Details, and profiles of the other pointings, are provided in English (1994).

\subsection{Optical Observations and Reductions}

On the night of 27-28 Feb 1995 we observed the Vela Cloud with the TAURUS imaging Fabry-Perot interferometer mounted at the $\mathrm{f} / 8$ Cassegrain focus of the AAT $3.9 \mathrm{~m}$.

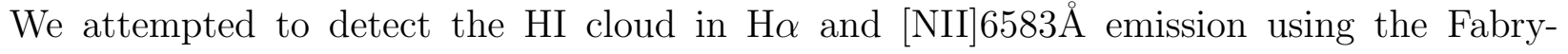
Perot 'staring' method which reaches the faintest possible levels in diffuse line emission (Bland-Hawthorn et al. 1994). The conditions were mostly photometric and dark, with the moon rising at the end of the night. We used the University of Maryland etalon with a 41 $\mu \mathrm{m}$ gap spacing and a coating finesse of 50 at $\mathrm{H} \alpha$. The blocking filter, which was centred at $6641 \AA$ with a $17 \AA$ bandpass, had a peak transmission of $73 \%$. The measured free spectral range was $54.3 \AA$ at $\mathrm{H} \alpha$ such that the blocking filter isolated only one order. We employed a TEK $1024^{2}$ CCD with 0.594 " pixels and a read noise of 2.3 electrons in XTRASLOW mode. The technique results in observations acquired in an annular region which was centred at $\alpha=10^{\mathrm{h}} 28^{\mathrm{m}} 30^{\mathrm{s}} \cdot 2, \delta=-44^{\circ} 08^{\prime} 51^{\prime \prime}(\mathrm{J} 2000)$. The integration times are listed in Table 2 ,

The images were bias subtracted, flatfielded and corrected for filter transmission profile using twilight and whitelight frames respectively. Stars within the field were located with daophot and subsequently subtracted. The four data frames where combined and co-added using minmax clipping under imcombine in IRAF; this procedure successfully removed all cosmic rays and point-like artefacts. The data were azimuthally binned and corrected for

the angular dispersion as discussed in Bland-Hawthorn et al. (1998). The final spectrum is dominated by two $\mathrm{OH}$ sky-lines at $6627.6 \AA$ and $6634.2 \AA$. No $\mathrm{H} \alpha$ or [NII] emission can be 
detected at the level of $\operatorname{EM}(\mathrm{H} \alpha)=60 \mathrm{mR}$ and $\operatorname{EM}([\mathrm{NII}])=30 \mathrm{mR}(3 \sigma)$. Note that at $10^{4} \mathrm{~K}$, $\operatorname{EM}(\mathrm{H} \alpha)=30 \mathrm{mR}$ is equivalent to a surface brightness of $2.0 \times 10^{-19} \mathrm{erg} \mathrm{cm}^{-2} \mathrm{~s}^{-1} \operatorname{arcsec}^{-2}$.

Using the Double-beam Spectrograph at the Siding Spring Observatory 2.3m telescope, a spectrum $(\S 4.2)$ was acquired at $\alpha=10^{\mathrm{h}} 28^{\mathrm{m}} 25 \mathrm{~s} .77, \delta=-44^{\circ} 16^{\prime} 17^{\prime \prime} .5$ (J2000), at the position of the HII emission-line galaxy WPV060. The wavelength range is 6400-6850 , allowing the acquisition of 5 emission lines: $\mathrm{H} \alpha$, [NII], [SII]. Using the $\sigma$ of the calibration arclamp lines gives an uncertainty for the systemic velocity of $6 \mathrm{~km} \mathrm{~s}^{-1}$. The instrumental profile is $4.8 \AA$ which corresponds to a velocity resolution of $\sim 220 \mathrm{~km} \mathrm{~s}^{-1}$. The reductions were carried out in the usual way using IRAF. For the spectrum and its analysis see $\S 4.2$.

\section{3. $21 \mathrm{~cm}$ Emission-line Measurement and Analysis Techniques}

As described in $\S$ 1.2, the Vela Cloud appears in our ATCA data as 3 large diffuse

components which we have labelled A, B, and C (Figure 2). This current section describes the techniques, including the uncertainties, used to measure the observed characteristics of these diffuse component clouds $(\S[3.1)$ and the enhancements embedded in them ( $\S 3.2)$. The measurements themselves are presented in section $\S 4$.

\subsection{The Diffuse Emission in Components A, B, and C}

For each diffuse cloud component sketched in Fig 2, the Full-Width-Half-Maximum (FWHM) value (§ 4.1) was determined from velocity profiles which were generated interactively using the KARMA visualization package (Gooch 1996). An uncertainty of 10-15\% includes the difference between measuring smoothed and unsmoothed profiles and the variation produced by visually selecting slightly different sized rectangles to encompass the component.

Dimensions and integrated flux densities were measured from integrated intensity (i.e. zero moment) maps generated using moment in MiRIAD. All measurements were made on data corrected for the primary beam response; however in the case of integrated intensity maps, the primary beam correction was applied to each moment map. Using cloud component $\mathrm{B}$, we compared this approach to constructing moment maps from primary beam corrected cubes. For these comparison maps, a smoothed mask cube was applied to a primary beam corrected cube and values above a $3 \sigma$ in the mask are retained and then a moment map was created from the resultant blanked cube. To create the mask cube, the primary beam corrected data were spatially smoothed using a Gaussian of 3 beam widths and Hanning 
smoothed in velocity using 3 channels (see Table 3). We note that the Vela Cloud is clearly evident in this mask cube. This second approach gave an integrated flux density for component B that was $14 \%$ higher than the value from the primary beam-corrected moment map approach. Since the primary beam-corrected moment map approach generates smaller values we list these for the integrated flux density, $\int \mathrm{S} \mathrm{dv}$ (in $\mathrm{Jy} \times \mathrm{km} \mathrm{s}^{-1}$ ), in Table 3 and in $\S 4.1$. The difference between measuring the flux density using moment maps (in which weak features may be clipped) and summing channel-by-channel (in which noise may also be summed) can be as large as 30\%, and we use this as our uncertainty. The uncertainty in the values of each component's dimensions is about $10 \%$ and reflects the difficulty in determining a component's boundary.

To calculate the HI mass in emission, $\mathcal{M}_{\mathrm{HI}}$, for each diffuse component we use the integrated flux density. To be consistent with the analysis of NGC 3256 in English et al. (2003), we use a distance of $37.6 \mathrm{Mpc}$, adopting $\mathrm{H}_{o}$ of $75 \mathrm{~km} \mathrm{~s}^{-1} \mathrm{Mpc}^{-1}$. (The resultant linear scale is $182 \mathrm{pc}$ per $1^{\prime \prime}$.) Combining the uncertainty in the flux density above with an uncertainty of $10 \%$ for the distance, increases the total uncertainty in the HI mass to at least $33 \%$.

The column density of each component, used for comparisons with HI clouds in other systems and objects within the NGC 3256 group (§ 6.1), is also listed in Table 3. For the polygonal area delineating each cloud component, we calculated the mean intensity $\mathrm{S}$ for the number of channels associated with the velocity range listed in Table 3 . However in order to calculate column density one must measure the brightness temperature $T_{B}$, rather than S. Using the Rayleigh-Jeans approximation,

$$
\mathrm{T}_{\mathrm{B}}=\frac{\lambda^{2} \mathrm{~B}}{2 \mathrm{k}}
$$

where $\mathrm{k}$ is the Boltzmann constant and $\mathrm{B}$ the brightness gives

$$
\mathrm{T}_{\mathrm{B}}=1541 \lambda(\mathrm{cm})^{2} \mathrm{~B}
$$

in Kelvin. The value for S, which is in $\mathrm{Jy} \mathrm{beam}^{-1}$, can be converted to B by describing the synthesized beam as a factor of $(\pi /(4 \ln 2))$ times the major and minor beam axes. Therefore, in terms of the measured parameters, the column density (e.g. equation 3 of Dickey \& Lockman 1990) becomes

$$
\mathrm{N}_{\mathrm{HI}}=1.823 \times 10^{18} \times \Delta \mathrm{v} \times \text { no. channels } \times \mathrm{S} \times \frac{1 \text { beam }}{1.1331 \theta_{\text {major }} \times \theta_{\text {minor }}} \times 1541 \times \lambda^{2}
$$

in atoms $\mathrm{cm}^{-2}$. The channel width $\Delta \mathrm{v}$, in $\mathrm{km} \mathrm{s}^{-1}$, and the axes of the synthesized beam $\theta$, in arcsec, are presented in Table 1, and the rest wavelength of the observations, $\lambda$, is 21 
$\mathrm{cm}$. An uncertainty of $<15 \%$ in the column density arises mainly from the selection of the number of channels associated with a given diffuse component.

The value of the peak (or maximum) column density within the total spatial region of each diffuse cloud component was determined from unclipped moment maps. Comparison with the clipped moment maps results in an uncertainty of about $4 \%$.

Note that the same techniques above were used to measure other objects in the dataset such as NGC 3263 and the apparent bridge between it and ESO 263-G044. For example, a moment map, constructed only using absolute values greater than $3 \mathrm{mJy} \mathrm{beam}^{-1}$, is used for the bridge's integrated flux density while both this map and a channel-by-channel calculation constrain its possible range of column density values ( $\$ 6.1)$.

\subsection{Detecting Enhancement Candidates Within Each Component}

There is evidence that Cloud B and the southern part of Cloud C host intensity enhancements. As an initial detection criterion, the potential HI enhancements were required to appear morphologically continuous in adjacent channels of the data cube. Additionally they have flux density peaks that are at least $6 \times$ r.m.s. in the HI cube.

In order to distinguish enhancements from random fluctuations in the diffuse components which are their hosts, we measured the mean flux density of the host $\left(3 \mathrm{mJy} \mathrm{beam}^{-1}\right.$ in both $\mathrm{B}$ and $\mathrm{C}$ ) and the host's r.m.s. (2 mJy beam ${ }^{-1}$ and $3 \mathrm{mJy} \mathrm{beam}^{-1}$ for $\mathrm{B}$ and $\mathrm{C}$ respectively). The enhancement was considered a robust candidate if its flux density peak was more than twice this r.m.s. above the mean flux density of their host component; this signal-to-noise is listed as "Peak Intensity r.m.s." in Table 4.

The Hanning smoothed (over 3 channels) velocity profiles for 3 of these enhancements are presented in $\S 4.2$. The spatial region of the enhancement was selected via a rectangle and its profile plotted for a velocity range larger than the range of the diffuse cloud component. We compared these profiles to those produced by encompassing the diffuse emission of a cloud component in a polygon which avoided the enhancements, confirming that the velocity range of diffuse gas was larger than the range of each enhancement. Also this comparison, along with visual examination of the cube, indicated which velocity peak in the profile is associated with each enhancement. The FWHM and the systemic velocity were measured for these narrower peaks using only the region of the profile above the host's mean emission value. The uncertainties in the enhancements' FWHM (a channel width, before smoothing, of about $6 \mathrm{~km} \mathrm{~s}^{-1}$ ) and central velocities reflect the uncertainty in visually selecting velocity features. Note that the $\mathrm{C} 1$ enhancement has 2 peaks that blend around the $50 \%$ level, so we 
do not quote a FWHM for this candidate. We adopt as its systemic velocity the channel in which C's main features appear simultaneously. These characteristics are also presented in $\S 4.2$ and listed in Table 4.

In order to measure the emission from an enhancement that does not include emission from its host, we first created subcubes spanning only the FWHM velocity range of the enhancement. We next subtracted from each channel of this cube the mean value of the emission due to the diffuse component of the cloud and subsequently constructed integrated intensity maps, using the 2 different approaches described in the previous section. The moment maps are presented in $\S 4.2$.

Since the enhancements are only slightly larger than the dimensions of the synthesized beam, the emission in each apparent enhancement was fit (using imfit in MIRIAD) with a Gaussian having the width of the point-spread-function of the synthesized beam. Using this fitting routine allowed us to avoid nearby emission that would be measured within visually selected polygons. With respect to uncertainties, we note that measuring B1 and C2 in integrated intensity maps formed from the blanked cube increases the flux density by only 1-3\%. Instead our uncertainty in the flux density of the enhancements, of less than 0.06 $\mathrm{Jy} \times \mathrm{km} \mathrm{s}^{-1}$, is estimated from the uncertainty in velocity (i.e. the selection of the number of channels for the subcube) plus the $7 \%$ increase that would occur if a polygon were used for the fitting. However we note that if the candidate enhancement's flux density should include the emission currently attributed to the host cloud, then its flux density could be larger by a factor up to 1.7 .

This integrated flux density was used to calculate the amount of HI mass in emission

of each enhancement (e.g. equation 8.24 in Binney \& Merrifield (1998)). Including the uncertainty in $\mathrm{H}_{o}$ generates an uncertainty in mass of $\leq 29 \%$.

Although the position of an enhancement is the average of a few different analysis techniques, to calculate its uncertainty we use the size of the synthesized beam divided by the signal-to-noise. This results in a mean uncertainty for the set of candidates of $26^{\prime \prime}$.

\section{Presentation of Measurements and Results}

The measurements, analysis and results presented in this section are used to consider star formation possibilities within the Vela Cloud $(\S[5)$, for comparisons of the Vela Cloud with other HI clouds ( $\S$ (6), and in the discussion of the origin and fate of the Vela Cloud (§ 7 ). 


\subsection{Tracing the Vela Cloud within the NGC 3256 Group}

\subsubsection{Radio data}

Fig. 1 shows The Vela Cloud's location in the NGC 3256 Group with the ATCA HI emission overlaid on the Digitized Sky Survey 1 . The velocity field of the NGC 3256 group and the Vela Cloud is shown in Fig. 3. These images roughly span $700 \mathrm{~km} \mathrm{~s}^{-1}$ and $39^{\prime} \times 43^{\prime}$ but do not show the whole group of galaxies.

NGC 3256C, NGC 3256, and NGC 3263 (labelled in Fig. 1) have clearly been disturbed by galaxy-galaxy interactions. Predominant in these data is NGC 3263, which we determine has an HI mass of $2 \times 10^{10} \mathcal{M}_{\odot}$. It is noticeable in Fig. 1 that the tidal tail of NGC 3263 extends to the east of that galaxy, while the Vela Cloud is projected onto the plane of the sky to the west of NGC 3263. In Fig. 3 NGC 3263's western velocity is quite distinct from that of the Vela Cloud. At NGC 3263's eastern side the velocity is $3309 \mathrm{~km} \mathrm{~s}^{-1}$ while its western side's velocity is $2668 \mathrm{~km} \mathrm{~s}^{-1}$. Indeed the Vela Cloud's velocity range of $2786 \mathrm{~km} \mathrm{~s}^{-1}$ to $2938 \mathrm{~km} \mathrm{~s}^{-1}$ (Table 3) is similar to the eastern tidal tail of NGC 3256.

Although they are usually not included in the Group in previous studies, we also consider ESO 263-G044 and ESO 263-G046 to be members of the NGC 3256 Group. The average of NGC 3263's systemic velocity $\left(2989 \mathrm{~km} \mathrm{~s}^{-1}\right)$ and that of NGC $3256\left(2820 \mathrm{~km} \mathrm{~s}^{-1}\right.$; English et al. 2003) is roughly $2900 \mathrm{~km} \mathrm{~s}^{-1}$, which we adopt for the moment to represent the central velocity for the group. The HI profiles of ESO 263-G044 and ESO 263-G046 give, with uncertainties less than a channel width, systemic velocities of $3064 \mathrm{~km} \mathrm{~s}^{-1}$ and 3067 $\mathrm{km} \mathrm{s}^{-1}$, respectively. Thus, assuming a moderate group velocity dispersion $\left(\leq 300 \mathrm{~km} \mathrm{~s}^{-1}\right)$, they are likely part of this system of galaxies.

This inclusion in the Group is supported by a possible bridge of HI that appears between NGC 3263 and ESO263-G044; please see Fig. 4. With respect to velocity continuity, the middle of the bridge appears in almost all channels from $3051 \mathrm{~km} \mathrm{~s}^{-1}$ to $3164 \mathrm{~km} \mathrm{~s}^{-1}$ while there is also an apparent trend for material to emanate from ESO263-G044 (around $\alpha=$ $\left.10^{\mathrm{h}} 29^{\mathrm{m}} 27.7^{\mathrm{s}}, \delta=-44^{\circ} 15^{\prime} 58^{\prime \prime}\right)$ at the lower velocities and attach to NGC 3263 at the higher velocities (around $\alpha=10^{\mathrm{h}} 29^{\mathrm{m}} 23^{\prime \prime} .1, \delta=-44^{\circ} 09^{\prime} 48^{\prime \prime}$ ). An integrated intensity map gives a

\footnotetext{
${ }^{1}$ Based on photographic data obtained using The UK Schmidt Telescope. The UK Schmidt Telescope was operated by the Royal Observatory Edinburgh, with funding from the UK Science and Engineering Research Council, until 1988 June, and thereafter by the Anglo-Australian Observatory. Original plate material is copyright the Royal Observatory Edinburgh and the Anglo-Australian Observatory. The plates were processed into the present compressed digital form with their permission. The Digitized Sky Survey was produced at the Space Telescope Science Institute under US Government grant NAG W-2166.
} 
conservative column density of $3 \times 10^{19}$ atoms $\mathrm{cm}^{-2}$ while a channel-by-channel calculation gives twice the value. The integrated flux density is $0.78 \mathrm{Jy} \times \mathrm{km} \mathrm{s}^{-1}$. In $\S$ 6.1, where we discuss the origin of the Vela Cloud, we note that this bridge is indicative of an interaction between NGC 3263 and ESO263-G044

With respect to the Vela Cloud itself, we have designated the most northerly structure component $\mathrm{A}$ and it arcs from about $\alpha=10^{\mathrm{h}} 28^{\mathrm{m}} 41^{\mathrm{s}}, \delta=-44^{\circ} 03^{\prime} 40^{\prime \prime}$ to $\alpha=10^{\mathrm{h}} 28^{\mathrm{m}} 18^{\mathrm{s}}$, $\delta=-44^{\circ} 03^{\prime} 50^{\prime \prime}(\mathrm{J} 2000)$, with a length of about $40 \mathrm{kpc}$. (We avoided measuring features to the east since they may be part of NGC 3263.)

While $\mathrm{A}$ is quite diffuse, component $\mathrm{B}$ appears to consist of a resolved amorphous structure enclosed in a larger diffuse elliptically shaped cloud. We refer to the more resolved structure as "interior" and the larger envelope as "exterior"; please see Fig. 2 and compare this to velocities 2892.0 and $2911.9 \mathrm{~km} \mathrm{~s}^{-1}$ in Fig. 6. The exterior structure is centred around $\alpha=10^{\mathrm{h}} 28^{\mathrm{m}} 28^{\mathrm{s}}, \delta=-44^{\circ} 08^{\prime} 40^{\prime \prime}$ and covers a region at least $67 \mathrm{kpc}$ wide. The interior structure contains 2 intensity enhancements.

Component $\mathrm{C}$ runs diagonally for almost $139 \mathrm{kpc}$ from northeast at $\alpha=10^{\mathrm{h}} 28^{\mathrm{m}} 50^{\mathrm{s}}$, $\delta=-44^{\circ} 08^{\prime} 30^{\prime \prime}$ (beginning at B's east most side) to the southwest at $\alpha=10^{\mathrm{h}} 28^{\mathrm{m}} 6^{\mathrm{s}}$, $\delta=-44^{\circ} 18^{\prime} 19^{\prime \prime}$. It's projected width is typically about $20 \mathrm{kpc}$ and spans $47 \mathrm{kpc}$ at its widest part. Component $\mathrm{C}$ also has 2 intensity enhancements. We do not report a FWHM for $\mathrm{C}$ since it has multiple velocity features.

The observed and derived characteristics of the diffuse components $\mathrm{A}, \mathrm{B}$, and $\mathrm{C}$ are given in Table 3, while the intensity enhancements are described in $\S$ 4.2. From Table 3 it can be noted that the full velocity range covered by the Vela Cloud is $152 \mathrm{~km} \mathrm{~s}^{-1}$ and each component's velocity dispersion, e.g. FWHM/2.35, is greater than the typical turbulent velocity $\left(10 \mathrm{~km} \mathrm{~s}^{-1}\right)$ in the Milky Way's ISM. The largest difference between the systemic velocities of the components is $61 \mathrm{~km} \mathrm{~s}^{-1}$. Although some portions of $\mathrm{C}$ are blueshifted relative to the other components, there are no velocity signatures that clearly indicate that the Vela Cloud, or any component of the Vela Cloud, is rotating, expanding or collapsing. Of course if our line of sight is perpendicular to the cloud's motion, we would not observe rotation or flows if these were occurring along the plane of the sky.

\subsubsection{Optical data}

In the $\mathrm{H} \alpha$ emission data from the SuperCosmos I Sky Survey (Hambly et al. 2001) the region of the Vela Cloud has the same statistical characteristics as the other empty regions of the sky. Outlined on Fig. 5 is the annular region observed with the TAURUS Fabry-Perot 
on the Anglo-Australian Telescope ( $\S 2.2)$. $\mathrm{H} \alpha$ emission was not detected in these data either. The $3 \sigma$ level non-detection corresponds to $30-60 \mathrm{mR}$, or a surface brightness on order of $10^{-19} \mathrm{erg} \mathrm{cm}^{-2} \mathrm{~s}^{-1}(\S 2.2)$, which indicates that the portion of the cloud within the annulus plotted on Component $\mathrm{B}$ is unlikely to be ionized by the UV radiation field of NGC 3263 or by strong shocks that would be created by colliding gas. Although the cosmic background does produce very weak levels of $\mathrm{H} \alpha$ emission (Bland-Hawthorn et al. 1994), we would have needed about 16 hours and photometric conditions to reach these depths using the Fabry-Perot staring technique.

Since this region was selected using preliminary $\mathrm{HI}$ data, more $\mathrm{H} \alpha$ observations would be required in order to cover other regions of the cloud; this is discussed in $\S 5.4$.

A preliminary search for starlight in the region of the Vela Cloud was undertaken with CCD imaging at the 40-inch Siding Spring telescope. The sky background in the V passband was $20.84 \mathrm{mag} \operatorname{arcsec}^{-2}$, while the peak in the cloud was $20.82 \mathrm{mag} \operatorname{arcsec}^{-2}$. This $2 \%$ increase in surface brightness corresponds to only $4 \sigma$ of the sky background so is not a significant detection. This image indicates that there is no evidence for starlight in the Vela Cloud at a surface brightness exceeding about $3 L_{\odot} \mathrm{pc}^{-2}$. Although the position of the Vela Cloud is at low Galactic latitude, supplemental observations at infrared wavelengths could prove helpful in confirming this.

\subsection{Delineating the Enhancements in the Vela Cloud}

We find 4 well-defined enhancements in the emission when analyzing the HI cube (Table1) using the criteria described in $\S$ 3.2. (That section also presents measurement methods and uncertainty estimates for measurements presented in this section.) Although each map in Fig. 6 combines 3 channels from the HI data cube, the enhancements in cloud component $\mathrm{C}$ are clear at $2792.7 \mathrm{~km} \mathrm{~s}^{-1}$ while the enhancements in component $\mathrm{B}$ are evident at $2892 \mathrm{~km} \mathrm{~s}^{-1}$. The enhancements are also evident at these velocities in the profiles ( $\S 3.2$ ) presented in Figure 7. These 3 profiles include some of the diffuse cloud emission which is spread over a broader velocity range (e.g. Table 3). However the width of the narrow peak, measured above the mean HI intensity of the host diffuse component, is consistent with each enhancement's velocity range as determined by visually examining the cube.

We are motivated to distinguish between the host's emission and the enhancement's emission in order to explore whether star formation occurs in the enhancement candidates in the Vela Cloud as it does in molecular density enhancements embedded in diffuse clouds $(\S$ 5.3). Thus we subtracted the mean flux density of each diffuse host component and 
created intensity maps integrated over the FWHM of the enhancements ( $\$ 3.2)$. The emission enhancements are clearly seen in these maps (Fig. 8). Their positions, labeled on Fig. 5, show that they do not coincide with 2MASS infra-red sources.

The observed characteristics of the enhancements are listed in Table 4. For example, the peak column density of each of the enhancements is on order of $10^{20}$ atoms $\mathrm{cm}^{-2}$. The listed integrated flux densities have been converted into $\mathcal{M}_{\mathrm{HI}}$. These $\mathrm{HI}$ emission masses are of order $10^{8} \mathcal{M}_{\odot}$ and are listed in Table 5 along with the enhancemnts' velocity dispersions, which are $\sigma_{\mathrm{o}}<20 \mathrm{~km} \mathrm{~s}^{-1}$.

C2 has the highest integrated flux density, and hence HI mass, and the largest velocity width of the 3 measured enhancements. Interestingly, projected onto the plane of the sky about 30" west of the peak of $\mathrm{C} 2$, and within this enhancement's bounds, is an HII galaxy, WPV060, cataloged by Winkler et al. (1994). Although barely resolved in SuperCosmos data, this galaxy (also known as 2MASX J10282575-4416172) has an estimated diameter of $7.4^{\prime \prime}$ for an isophotal of $20.0 \mathrm{~K}-\mathrm{mag} \operatorname{arcsec}^{-2}$ (NED)2. However the following comparison of its optical spectrum (described in $\S 2.2$ ) with ATCA HI emission shows that WPV060 does not appear, at least at the resolution scales of the HI cube listed in Table 1, to be associated with the HI of enhancement $\mathrm{C} 2$.

The heliocentric velocity of WPV060, as measured from 5 emission lines ( $\mathrm{H} \alpha$, [NII], [SII]: see Fig. 9) in our optical spectrum, is $2675 \pm 6 \mathrm{~km} \mathrm{~s}^{-1}$ (§ 2.2), while the HI profile of $\mathrm{C} 2$ peaks at $2815 \pm 3 \mathrm{~km}^{-1}$. The emission lines are unresolved by the optical spectrometer. The integrated HI intensity map ( Fig. 9) shows no significant HI emission associated with WPV060 within $\pm 50 \mathrm{~km}^{-1}$ of its optical systemic velocity. While WPV060 is likely to be a member of the NGC 3256 group, we conclude that it and C2 are probably not directly related.

\section{Derived Characteristics Relevant to Forming Star Clusters}

We wish to explore the Vela Cloud's potential to form stars within the candidate density enhancements. Therefore in this section we focus on deriving mass and volume estimates, which in turn constrain values of density and pressure. We consider a scenario in which the cloud components are in pressure equilibrium and, if so, assess whether the enhancements

\footnotetext{
${ }^{2}$ This research has made use of the NASA/IPAC Extragalactic Database (NED) which is operated by the Jet Propulsion Laboratory, California Institute of Technology, under contract with the National Aeronautics and Space Administration.
} 
may collapse to form stars by representing them as Bonnor-Ebert spheres.

\subsection{HI Mass}

The total HI mass estimate for the Vela Cloud $-[3$ to 5$] \times 10^{9} \mathcal{M}_{\odot}-$ is on order of the HI content of normal Sc galaxies (e.g. Bothun \& Sullivan 1980). The lower limit for this estimate comes from our analysis of the ATCA HI cube (Table 1). That is, the sum of the integrated flux densities of the 3 diffuse components (Table 3), which is $10 \pm 3 \mathrm{Jy}$ $\mathrm{km} \mathrm{s}^{-1}$, shows that the cloud contains at least $3 \times 10^{9} \mathcal{M}_{\odot}$, with an uncertainty of $33 \%$ ( $§ 3.1$. For the upper limit, we use the Parkes Telescope single-dish data to compensate for the emission not detected by an interferometer. The Vela Cloud was detected in 3 pointings (English 1994) and Fig. 10 shows the velocity profile at the pointing $\alpha=10^{\mathrm{h}} 28^{\mathrm{m}} 27^{\mathrm{s}} .7$, $\delta=-44^{\circ} 09^{\prime} 26^{\prime \prime}(\mathrm{J} 2000)$. Comparison of the Parkes profiles with the ATCA data indicates that the narrow Gaussian component in the profile is associated with the Vela Cloud, rather than NGC 3263. Using the area under the peak of this component in Fig. 10, between 2760 and $2975 \mathrm{~km} \mathrm{~s}^{-1}$, generates an upper limit for the total emission of $15 \pm 2 \mathrm{Jy} \mathrm{km} \mathrm{s}$ and a corresponding HI mass for the cloud of about $5 \times 10^{9} \mathcal{M}_{\odot}$. The mass estimate is also comparable to the combined mass associated with HI emission in both tails of NGC 3256, $\sim 4.7 \times 10^{9} \mathcal{M}_{\odot}($ English et al. 2003$)$.

The mass in HI emission for each enhancement alone, listed in Table 5, is about 1 $\times 10^{8}$. (Recall that this estimate is conservative since we subtracted emission which could be attributed to the diffuse host cloud.) English et al. (2003) argue that, if star formation has only $10 \%$ efficiency, the amount of gas required in order to form massive clusters of stars, such as globular clusters, is on the order of $10^{7 \pm 1} \mathcal{M}_{\odot}$. Thus the enhancements' HI mass is at the high end of this mass range.

\subsection{Pressure Equilibrium}

\subsubsection{Deriving Pressure}

The pressure of the diffuse HI gas component, required for our Bonnor-Ebert sphere analysis in the following section, can be determined from

$$
\mathrm{P}=\rho \sigma^{2}
$$

where $\rho$ is the volume density and $\sigma$ is the velocity dispersion. Although the mean density

of a region of atomic hydrogen is simply $\rho=\mathcal{M}_{\mathrm{HI}} /$ volume, we need to adopt a spatial scale 
along the line of sight (LOS).

We measure the pressure in the diffuse gas of $\mathrm{A}$ and $\mathrm{C}$ by selecting in each component a representative rectangular area, avoiding enhancement candidates. In component $\mathrm{C}$, we additionally avoid the diagonal structure at redshifts $\geq 2825 \mathrm{~km} \mathrm{~s}^{-1}$. For the LOS axis we took the average of the region's length (e.g. major axis) and width (e.g. minor axis). The region in $\mathrm{C}$ is $20 \mathrm{kpc} \times 16 \mathrm{kpc}$, encloses $1.06 \times 10^{8} \mathcal{M}_{\odot}$ of $\mathrm{HI}$, has an uncertainty in the LOS axis of $9 \%$, the standard deviation in $\sigma$ is $11 \%$, and the combined uncertainty in pressure is $40 \%$. In component $\mathrm{A}$ the region is $26 \mathrm{kpc} \times 49 \mathrm{kpc}$, contains $1.56 \times 10^{7} \mathcal{M}_{\odot}$ of $\mathrm{HI}$, and the uncertainty in the LOS axis is $24 \%$. The uncertainty in A's pressure may be larger than $50 \%$ which is indicated by the difference between the velocity range determined by visual inspection of the HI cube and the FWHM measured from the intensity profiles ( $\$ 3.1)$.

Cloud component B is more complex. $\S 4.1 .1$ describes the interior and exterior structures of B (also see Fig. 2) and the values of the parameters used to derive mass and pressure are presented in Table 3, For each structure in B we calculate the pressure for two simple models. One is that of an oblate-shaped cloud (i.e. half the length of the LOS axis = the semi-major axis) and the other for an prolate-shaped cloud (i.e. half the length of the LOS axis $=$ semi-minor axis). We then average these estimates together. To determine the mass and volume of the exterior component alone, the mass and volume of the interior component of $B$ were subtracted from those values for the whole cloud. Combining the uncertainties from the flux density (i.e. the dominant uncertainty), the measurement of the axes (large for the LOS axis), the FWHM, and the distance, there is an uncertainty in each structure's pressure of about $43 \%$.

The pressure $(\mathrm{P} / \mathrm{k})$ of each diffuse component is presented in Table 3, They are normalized by the Boltzmann constant for comparison with pressures in theoretical studies. These pressures, which range from 5 to $100 \mathrm{~K} \mathrm{~cm}^{-3}$, are used in $\S 5.3$ where we calculate the Bonnor-Ebert mass of each enhancement candidate.

\subsubsection{Pressure Equilibrium Discussion}

If the diffuse part of the cloud were in pressure equilibrium, then we could employ virial theorem arguments to estimate the masses (independent of the mass in HI emission) required if the enhancements are about to form stars $\left(\S\left[\begin{array}{l}5.3 \\ \text { ) }\end{array}\right.\right.$. As pointed out in $\S$ 4.1, component B appears to consist of a resolved structure interior to a more diffuse, exterior shell; see Fig. 2 , This is analogous to the well-explored theoretical scenario of a molecular cloud surrounded by the ISM's HI. Applying the analogy to our data, if the pressure in the interior HI structure 
is at least equal to that in the exterior HI shell then these 2 structures could be in pressure equilibrium. Although the pressures of these structures, listed in Table 3, differ by $40 \%$, the error in the estimate of the exterior pressure is at least $43 \%$ ( $\S 5.2 .1$ ). Therefore we can consider the possibility that the interior and exterior diffuse structures are in pressure equilibrium and use their average as the value for the ISM's pressure in cloud component B.

Although we then assume that the Vela Cloud is in equilibrium for subsequent calculations, the clumps and irregular morphology indicate that this may not be the case. A dynamical crossing time in component $\mathrm{B}$, estimated using the radius divided by the velocity dispersion, is 1-2 Gyr. If this is a primordial cloud then there has been sufficient time for

virialization. However the age estimate of the tidal tails of NGC 3256 (English et al. 2003) is 500 Myrs. Hence the cloud may not yet be in equilibrium if the cloud is the remnant of the galaxy-galaxy interactions which also precipitated the formation of NGC 3256.

\subsection{Bonnor-Ebert Mass and Star Formation in the Enhancements}

Again applying the molecular cloud analogy to our data, the enhancements, which appear to be distinct velocity features in velocity profiles (Figure 7), would be analogous to molecular cores. In this section we consider whether, given the conditions in the surrounding diffuse gas, there is sufficient mass in the enhancements to cause star formation. This analysis uses the velocity dispersion of each enhancement $\left(\sigma_{o}\right)$ and the pressure of its host diffuse component. That is, we assume that the enhancements are in pressure equilibrium with the $\mathrm{HI}$ in which they are embedded and hence their surface pressures $\left(\mathrm{P}_{\mathrm{s}}\right)$ are equivalent to the pressures in the diffuse cloud components. The pressure is $\mathrm{P} / \mathrm{k} \sim 72 \mathrm{~K} \mathrm{~cm}^{-3}$ for the diffuse component $\mathrm{B}(\S 5.2 .2)$ and $\mathrm{P} / \mathrm{k} \sim 39 \mathrm{~K} \mathrm{~cm}^{-3}$ for component $\mathrm{C}$ (Table 3). Including uncertainties in their measurements, the pressure in $\mathrm{B}$ is $\mathrm{P} / \mathrm{k} \sim 32-100 \mathrm{~K} \mathrm{~cm}^{-3}$ and $\mathrm{C}$ has $\mathrm{P} / \mathrm{k} \sim 23-55 \mathrm{~K} \mathrm{~cm}^{-3}$. Although each enhancement contains a sufficient $\mathrm{HI}$ gas mass to populate a globular cluster if it were converted to stars, these pressures are far short of the $\mathrm{P} / \mathrm{k}=10^{6}-10^{8} \mathrm{~K} \mathrm{~cm}^{-3}$ proposed by Elmegreen \& Efremov (1997) as a requirement for the formation of such massive, bound structures.

However we can calculate, using the given pressure and velocity conditions, the amount of mass that would be required by each enhancement in order for it to form stars, regardless of whether they are in a cluster. To do so we assume that each embedded enhancement is an isothermal sphere on the verge of collapse (i.e. a Bonnor-Ebert sphere). We note that the enhancements, which are slightly larger than the synthesized beam, may be approximated by spheres in these data (e.g. Figure 8). Bonnor (1956) defines the radius of the interface 
of an isothermal sphere with the ISM as

$$
r_{c}=0.49\left(\frac{k T}{m G \rho_{c}}\right)^{1 / 2}
$$

where $\mathrm{m}$ is the molecular weight and $\rho_{\mathrm{c}}$, the critical density, is the density of the sphere also at the interface. The relationship between the mass within this radius and $r_{c}$ is

$$
M\left(r_{c}\right)=2.4 \frac{k T}{m G} r_{c}
$$

Substituting the first equation into the second, and using $\rho_{\mathrm{c}}=\mathrm{P}_{\mathrm{s}} / \sigma_{\mathrm{o}}^{2}$, and $\sigma_{\mathrm{o}}^{2}=\mathrm{k} \mathrm{T} / \mathrm{m}$, generates

$$
M\left(r_{c}\right)=1.18 \frac{\sigma_{o}^{4}}{G^{3 / 2} P_{s}^{1 / 2}}
$$

where $\sigma_{\mathrm{o}}$ is the velocity dispersion of the enhancement (i.e. FWHM of the enhancement in Table 4 divided by 2.35) and $\mathrm{P}_{\mathrm{s}}=\mathrm{P}$ of the ISM in the host component. If we had used $\sigma_{o}^{2} \times r_{c} \times G^{-1}$ for the virial mass, we would be forced to adopt the beam size as an upper limit for the unknown diameter. Thus it is noteworthy that the formulation above eliminates the need to measure the intrinsic radius of an enhancement.

These derived masses are listed in Table 5. Combining the largest uncertainties in $\mathrm{P}_{\mathrm{s}}$ and $\sigma_{\mathrm{o}}$ generates an uncertainty in the Bonnor-Ebert mass of about $40 \%$. This table also lists the ratio of the estimated Bonnor-Ebert mass to the HI mass in emission. Accounting for the mass range given by the uncertainties, this ratio would need to be $\frac{\mathcal{M}\left(\mathrm{r}_{\mathrm{c}}\right)}{\mathcal{M}_{\mathrm{HI}}} \leq 2.2$ for the HI mass to be equivalent to the Bonnor-Ebert mass, and hence star formation to be a possibility. However the $M\left(r_{c}\right)$ are 9-44 times larger than $\mathcal{M}_{\mathrm{HI}}$. We note that the HI mass measurement is a lower limit since we subtracted off a threshold that we assumed to be associated with the host cloud emission. Therefore if some of the emission attributed to the host belongs to the enhancements, then the HI enhancement candidates could have almost twice as much emission ( $\S 3.2$ ) than listed. Taking into account that this assumption also increases the FWHM, the mass ratios would then be about 6-22.

The analysis above indicates that if these enhancements are isothermal spheres composed solely of atomic hydrogen gas there is insufficient gas for star formation to occur. Using the values listed in Table 5, the amount of gas is deficient by about an order of magnitude; alternatively, the velocities within the enhancements are about 2 times too fast given the pressure of the surrounding medium. Another perspective is that the external pressure would need to increase in order to cause the enhancements to collapse and form stars. The increase in external pressure required is at least a factor of 9 and possibly as large as a factor of 400. The former estimate is based on B2 and attributes all of the observed emission to the enhancement, while the latter factor uses the tabulated HI mass estimate for C2. 


\subsection{The Ultra-Violet Radiation Field of NGC 3263}

The non-detection of ionized hydrogen noted in $\S 4.1 .2$ indicates that the portion within the TAURUS annulus is unlikely to be ionized by NGC 3263. In the current section we explore whether other regions of the Vela Cloud could nevertheless be ionized.

We have computed the ionizing radiation field from NGC 3263 by scaling up the Galaxy model of Bland-Hawthorn \& Maloney $(1999 ; 2002)$ by the ratio of the total blue luminosities, such that $\mathrm{L}_{\mathrm{B} \text { (NGC3263) }} / \mathrm{L}_{\mathrm{B} \text { (Galaxy) }}=10$. Without a detailed knowledge of the disk star formation history in this highly inclined galaxy, $\mathrm{L}_{\mathrm{B}}$ is a useful surrogate. (The measured ratio, $\sim 5$, has been doubled in order to generate a face-on model.) In our model, we adopt a total disk diameter of $20 \mathrm{kpc}$ and a mean disk opacity of $\tau_{L L}=2.8$ where $\tau_{L L}$ is the opacity at the Lyman limit.

In Fig. 11, we show the distribution of ionizing radiation above and below the galaxy plane. The uniform dust distribution in the disk elongates the radiation field along the minor axis. The contours show lines of constant ionizing flux. These can be converted to the expected emission measure in $\mathrm{H} \alpha$ with the following formula (Bland-Hawthorn \& Maloney 1999)

$$
\log (E M)=\log \phi-3.38
$$

where the emission measure $E M$ is given in milliRayleighs. The contours in Fig. 11] are given in units of $\log \phi$. Thus, at a distance of $80 \mathrm{kpc}$ off the plane, the predicted ionizing flux is $\log \phi=5$. This is sufficient to excite an HI cloud to an emission measure of about 400 $\mathrm{mR}$ which can be detected in a deep exposure with a tunable imaging filter (e.g. Veilleux et al. 2004). The Fabry-Perot staring method is able to improve on this by more than an order of magnitude (Bland-Hawthorn et al. 1994) which means that most of the HI even far from the spin axis of the galaxy (where $\log \phi>4.5$ ) would be ionized and rendered visible to this technique. Assuming the Vela Cloud is at the same distance as NGC 3263, if this is found not to be true, then the blue stellar population of NGC 3263 may be somewhat older than the Galactic population today, as is seen in M33, for example (Patel \& Wilson 1995). Alternatively our argument could be affected by uncertainty in the NGC 3263 model due to scaling up the Milky Way model. Additionally a non-detection could be explained by the strong dependence of the radiation field with polar angle (Bland-Hawthorn \& Maloney

1999). Another possibility is that the covering fraction of the gas is low as seen from the central galaxy.

However if our model is appropriate, the northern section of component $\mathrm{C}$ lies long the 
$\log \phi=5$ contour. It has a column density of $4.58 \times 10^{19}$ atoms $\mathrm{cm}^{-2}$ which is almost half that of C's southern section. Although observationally challenging, ionized emission could be detected from gas with this modest column density if the Vela Cloud is at the distance of NGC 3263.

\section{Comparison of The Vela Cloud with Other Clouds}

\subsection{The Vela Cloud's Diffuse Components and the NGC 3256 Group}

Summarizing the characteristics in Table 3, the Vela Cloud's column density range is $10^{19-20}$ atoms $\mathrm{cm}^{-2}$ and $\mathrm{HI}$ mass range is $10^{8-9} \mathcal{M}_{\odot}$. Although it is tempting to compare the Vela Cloud with numerous examples of HI tidal tails and plumes associated with galaxygalaxy interactions and mergers, the Vela Cloud is morphologically less contiguous and less

regular in velocity than even very unusual kinds of tidal debris (e.g. IC 2554 (Koribalski et al. 2003)). The Vela Cloud is most similar to the debris in clusters of galaxies, such as the plume near NGC 4388 and the HI tail from NGC 4254 in which the putative dark galaxy VirgoHI 21 (e.g. Minchin et al. (2007)) is embedded. The plume near NGC 4388 is $110 \times 25 \mathrm{kpc}$, has a few $\times 10^{8}$ solar masses of HI, a column density of about $10^{20}$ atoms cm $\mathrm{cm}^{-2}$ and a typical FWHM of $100 \mathrm{~km} \mathrm{~s}^{-1}$ (Oosterloo \& van Gorkom 2005). In the ALFALFA data, NGC 4254's tail has $6 \times 10^{8} \mathcal{M}_{\odot}$ and an extent of roughly $250 \mathrm{kpc}$ (Haynes et al. 2007).

With respect to apparently isolated clouds, as pointed out by Ryder et al. (2001), the Vela Cloud has a similar mass and extent as HIPASS J0731-69. However the Vela Cloud is larger than FCC 35's HI companion cloud (Putman et al. 1998) and the HIJASS J1021+6842 cloud in the M82 Group (Walter et al. 2005). Since these isolated clouds are dissimilar from each other, we cannot draw strong conclusions about the Vela Cloud based on these comparisons. Therefore, we focus on comparing the column density of the diffuse components of the Vela Cloud with other objects in the field of view of our HI data.

A possible bridge of HI material appears between NGC 3263 and ESO263-G044 ( $\$$ 4.1.1. Fig. (4). The length of the bridge is about $67 \mathrm{kpc}$ and the column density is $3-6 \times$ $10^{19}$ atoms $\mathrm{cm}^{-2}$. Thus the bridge's column density is similar to the Vela Cloud's diffuse components. The integrated flux density corresponds to HI mass of roughly $7 \times 10^{6} \mathcal{M}_{\odot}$, which is lower than component A by an order of magnitude.

While the combined mass in both tails of NGC 3256 is comparable to the total mass in the Vela Cloud, the eastern tidal tail's column density, $1.6 \times 10^{20}$ atoms $\mathrm{cm}^{-2}$ is larger than that of any individual component in the Vela Cloud. The value for the tail is determined from the HI cube presented in the current paper and has an uncertainty of $5 \%$. Note that 
the fragment north of NGC 3256 (called Fragment A2 in English et al. 2003) has a column density, in the current data, of $1.7 \times 10^{19}$ atoms $\mathrm{cm}^{-2}$, similar to component A.

\subsection{The Vela Cloud's Enhancements Versus Apparently Isolated Clouds}

The Vela Cloud's few enhancement candidates have a peak column density on the order of $10^{20}$ atoms $\mathrm{cm}^{-2}$, masses of about $10^{8} \mathcal{M}_{\odot}, \mathrm{FWHM}$ of 20 to $44 \mathrm{~km} \mathrm{~s}^{-1}$, and diameters of about $14 \mathrm{kpc}$ (see Table 4, Table 3, and Table 5). Although there are observational limits due to different angular resolutions (noted at the end of this section), we contrast these enhancements with observations of various kinds of HI clouds to see if similarities or differences can help illuminate the Vela Cloud's origin or fate ( $\S$ 7.2). Unfortunately the distance to some comparison clouds are unknown so we can only compare column densities and not physical parameters such as mass and diameter.

In general we find that the Vela Cloud's enhancements cannot easily be categorized with isolated HI clouds but instead differ from them in a variety of ways. For example the clumps in HIPASS J0731-69 each have almost as much HI mass as the Vela Cloud's enhancements $\left(\sim 5 \times 10^{7} \mathcal{M}_{\odot}\right.$; Rvder \& Koribalski (2004) $)$ yet the peak column density is less $\left(1.3 \times 10^{19}\right.$ atoms $\mathrm{cm}^{-2}$; Ryder et al. (2001)). The peak column density is also less in the HI companion to FCC $35\left(2.0 \times 10^{19}\right.$ atoms $\mathrm{cm}^{-2}$; Putman et al. (1998)). The HI companions to HII galaxies detected by Taylor et al. $(1993,1995)$ have some characteristics that are similar to those of the Vela Cloud enhancements; peak column densities ranging from $10^{19}$ to $10^{21}$ atoms $\mathrm{cm}^{-2}$, HI masses from $10^{8}$ to $10^{10} \mathcal{M}_{\odot}$ and diameters as small as a few kpc to a few tens of kpc. However, the companions' Half-Width-Half-Maximum range from 30 to $240 \mathrm{~km} \mathrm{~s}^{-1}$ while the Vela Cloud's enhancements' $\sigma_{\mathrm{o}}$ 's range from 13 to $19 \mathrm{~km} \mathrm{~s}^{-1}$. However there are optically unseen clouds in the Virgo cluster which have roughly $10^{8} \mathcal{M}_{\odot}$ and one cloud in particular consists of 5 clumps strewn across $200 \mathrm{kpc}$ and a few clumps have FWHMs of 50-60 $\mathrm{km} \mathrm{s}^{-1}$ (Kent 2008).

Convincing detections of stellar populations in HVCs associated with our Milky Way Galaxy remain elusive (e.g. Schneider et al. 1983, Willman et al. 2002, Simon \& Blitz 2003, Simon et al. 2006). Therefore HVCs are the most nearby HI clouds available for comparison with the enhancements in the Vela Cloud. The FWHMs of compact HVCs (CHVCs) are typically 25-35 $\mathrm{km} \mathrm{s}^{-1}$ (de Heij et al. 2002a). While the peak column densities of CHVCs are typically $10^{19} \mathrm{~cm}^{-2}$, an order of magnitude less than the Vela Cloud's enhancements, some rare CHVCs have peaks of $10^{20} \mathrm{~cm}^{-2}$ (Putman et al. 2002). The angular size of the compact variety is typically of $\sim 50$ arcmin (de Heij et al. 2002b). If CHVCs are primordial clouds falling into the potential of the Local Group of galaxies from a distance, say, of $1 \mathrm{Mpc}$, 
this may correspond to a linear diameter of of $15 \mathrm{kpc}$, similar to the enhancements. Under this condition the Vela Cloud's enhancements could be similar to the higher density compact HVCs. However distances to some HVC indicate that the population resides within tens of kpc from the Galactic plane (e.g. Kalberla \& Haud 2006 and references mentioned within; Wakker et al. 2008) and hence most HCVs would be much smaller than the enhancements.

Some non-compact HVCs have density enhancements surrounded by HI with column densities around $\sim 2 \times 10^{19} \mathrm{~cm}^{-2}$ (de Heij et al. 2002b, Kalberla \& Haud 2006), which is comparable to the column density of the surrounding diffuse component of the Vela Cloud. So we can consider whether the diffuse major components of the Vela Cloud are similar to these HVC. Peak column densities in HVC complexes can be on order of $\sim 10^{20} \mathrm{~cm}^{-2}$, e.g. complex A (Wakker \& Schwarz 1991) and the HIPASS HVCs (Putman et al. 2002). However these particular HVCs have typical linewidths of only $35 \mathrm{~km} \mathrm{~s}^{-1}$, very unlike the $97 \mathrm{~km} \mathrm{~s}^{-1}$ FWHM of cloud component B. As a specific example, Complex A's envelope has a linewidth of about $25 \mathrm{~km} \mathrm{~s}^{-1}$, the FWHM of its cores is only $10 \mathrm{~km} \mathrm{~s}^{-1}$ (Wakker \& van Woerden 1997), and its mass is only $10^{5-6} \mathcal{M}_{\odot}$ (van Woerden et al. 1999). Work by Kalberla \& Haud (2006) find the cores of the HVC typically have velocity dispersions of a few $\mathrm{km} \mathrm{s}^{-1}$, although the $\sigma_{o}>10 \mathrm{~km} \mathrm{~s}^{-1}$ of the Vela Cloud enhancements does not appear excluded. However they show that the envelops in HVC complexes have velocity dispersions of about $12 \mathrm{~km} \mathrm{~s}^{-1}$, compared to our estimate (FWHM/2.35) of $41 \mathrm{~km} \mathrm{~s}^{-1}$ for component B. Thus the diffuse components, in particular, of the Vela Cloud are evidently dissimilar to the envelopes of the HVC complexes.

We note that there are caveats to our comparisons. The use of different beam sizes and velocity resolutions can play a role in generating discrepancies when comparing extragalactic and Galactic clouds. The observational parameters are also convolved with different physical scales since the clouds are at different distances. Measurements of FWHM are problematic due to, for example, multiple peaks in the profiles and beam smearing of the velocity gradient. Additionally peak column densities are defined and measured differently in different papers. Nevertheless by doing these comparisons we find that the Vela Cloud, rather than clearly falling into a specific category of extragalactic HI cloud, instead contributes to the variety of types of extragalactic HI clouds. Also the Vela Cloud's enhancements may be similar only to CHVC and then only in the unlikely case that CHVC are at large distances from the Milky Way. 


\section{Discussion}

\subsection{Origin of the Vela Cloud}

The Vela Cloud, as delineated by our ATCA data, contains a few $\times 10^{9} \mathcal{M}_{\odot}$ of HI which is comparable to that in an Sc galaxy. However if this cloud contains stars or ionized hydrogen gas, these components are not obvious in our optical data. Therefore, since galaxy formation is not expected to be $100 \%$ efficient, the possibility exists that the Vela Cloud is a primordial cloud, an example of a building block in hierarchical galaxy formation scenario. Although various extragalactic clouds have been detected and characterized, the rarity of bona fide primordial clouds (\$1.1) means that their morphology, column density, and velocity behaviour is unknown. Thus primordial cloud characteristics can not be used to support this origin for the Vela Cloud.

Since the Vela Cloud resides in a group environment in which most galaxies show tidal disturbances (e.g. Fig.1), it is more likely that the Vela Cloud's origin is also tidal. A few properties of the Vela Cloud also suggest a tidal origin. For example, although the Vela Cloud is more tenuous, its total mass is similar to the amount in the tidal tails of NGC 3256. Also its column density is similar to NGC 3256's fragment A2 (§6.1). Therefore the intention of this section is to review a few relevant interaction scenarios, in light of the properties of the Vela Cloud and the Vela Cloud's environment, in order to assess which of these would be most relevant to the Vela Cloud's origin.

Bekki et al. (2005b) have numerically studied the effect on individual gas-rich galaxies due to the gravitational potential of a group of galaxies. Their model of a low surface brightness late-type galaxy orbiting the group's centre generates a starless Leo ring-type of HI structure within about 6 Gyr. The outer disk gas, which in this galaxy extends 5 times that of the stellar disk, is stripped off of the parent galaxy and enroute, at $\sim 4$ Gyr, the tidal structure is similar to that of HIPASS J0731-69. The observed morphology is the result of the range of HI column densities. That is, the structure is contiguous but with an inhomogeneous density such that only high density regions of the tidal arcs are revealed using current radio arrays. This scenario is appealing since qualitatively the structure at 4 Gyr is suggestive of the morphology of NGC 3256 combined with the Vela Cloud (compare our Fig 1 and Fig 3 to Fig. 1 in Bekki et al. (2005b)). However this picture is probably misleading for a number of reasons. In the model the parent galaxy does not form stellar tidal tails, which NGC 3256 does exhibit (e.g. Fig:1). Also the observed morphological features of NGC 3256 can be more simply accounted for by a major merger of 2 galaxies (e.g. English et al. 2003). Additionally the model shows that the parent galaxy resides outside the radius of the resultant ring, implying that galaxies other than NGC 3256 could 
have provided the Vela Cloud's gas instead.

A drawback of this group potential simulation is that it is particular about the characteristics of the parent galaxy as well as the distance from the centre of the group potential. That is, star formation will occur in the arcs drawn from a more massive galaxy and rings may not form if a high surface brightness or compact galaxy interacts with the group potential. We can relax these parent galaxy effects by using an analogy with clusters of galaxies since we expect the tidal forces in groups of galaxies to be analogous to those in clusters, although more minor in affect. We are also motivated by our assessment that the type of object that the Vela Cloud most resembles is debris within clusters ( $\S[6.1)$.

As galaxies orbit in a cluster environment, they are not only subject to the cluster potential itself, but also to interactions with individual galaxies. Take, for example, pairs of galaxies that interact in the outer regions of the cluster as they fall in (e.g. Dubinski 1998). Although tidal material pulled from the outer disk of each galaxy remains bound to an interacting system if the interaction occurs in the field, within a cluster environment these tails are also subject to the tidal field of the cluster. This causes the tails to be more extended and diffuse. It also efficiently strips the tail material out of the galactic potential, dispersing it throughout the cluster (Mihos 2004b, Mihos 2004a and papers mentioned therein; Bekki et al. 2005a).

In the case of the NGC 3256 group, most of the galaxies have peculiar morphologies that are similar to those in isolated interactions (e.g. Fig.1). None are so pathological that they require a mass like the Vela Cloud to exist in order to explain their features. As previously mentioned, NGC 3256 has the morphology of an on-going merger of 2-3 galaxies (e.g. English et al. 2003, Lípari et al. 2000). Another example is NGC 3263 which has an extended arm ( $\S$ 4.1.1) that can be generated when a smaller galaxy has an inclined orbit to a larger one (e.g. Quinn \& Goodman 1986). A candidate satellite galaxy can even be identified in our data; ESO263-G044 appears related to NGC 3263 by an HI bridge (\$ 4.1.1, $\S$ 6.1). Indeed NGC 3263's arm extends in the opposite direction to the Vela Cloud (e.g. Fig 3 , $\S 4.1 .1$ ), confirming that the Vela Cloud is superfluous for producing the tidal features of NGC 3263.

The apparent simplicity of these interacting systems suggests we should examine a scenario for the formation of the Vela Cloud which is analogous to the process described for interactions in clusters. Originally a typical interaction creates tidal features as the interacting pair falls into the group's potential. The tidal field of the group causes these features to be more extended and diffuse than they would be in the field. The group potential subsequently strips the tidal features off of the parent. Thus the Vela Cloud could be stripped tidal features. Additionally, since the tidal features, and hence much of the interacting galax- 
ies' HI gas, are removed in this process, it is difficult to recognize, by its morphology alone, any particular peculiar galaxy in the group as being the specific victim of an appropriate encounter. There are a number of candidates, within the large region of the association of galaxies, which are undetected in HI (e.g. ESO263-G045, 263-G8, 317-G4, N3250B, N3318A, 264-G14 (Mould et al. 1991)). However if we assume that the process that created the Vela Cloud occurred roughly when the tails of NGC 3256 were generated, then the most likely candidate is the gas-poor peculiar galaxy NGC 3256c. The projected distance between NGC 3256c and the Vela Cloud, and their velocity difference, implies that they were in close proximity roughly $600 \mathrm{Myr}$ ago while the timescale for NGC 3256's pericentre encounter was 500 Myr ago (English et al. 2003).

An alternative origin would be ram pressure stripping by the putative hot gas between the galaxies in the NGC 3256 Group. The situation would be similar to the case of the structure containing VirgoHI 21, which does have properties similar to the Vela Cloud (§6.1). VirgoHI 21 was considered to be an example of ram pressure stripping by Oosterloo \& Van Gorkom (2005). In this case one would be tempted to interpret the vertical extensions from NGC 3263, such as the "bridge", and the Vela Cloud as stripped gas. However the Oosterloo \& Van Gorkom argument was based on the observation that VirgoHI 21's parent galaxy NGC 4254 had a truncated HI disk. Since the disk of NGC 3263 is gas rich and extended, this instead suggests that a galaxy such as NGC 3256c would have been the victim of ram pressure stripping. Unfortunately for the ram pressure scenario recent, more sensitive observations show that VirgoHI 21 is embedded in an extensive HI plume from NGC 4254 (Haynes et al. 2007) and this plume has been successfully modelled as a tidal tail that was expelled from NGC 4254 during the flyby of a massive companion galaxy (Duc \& Bournaud 2008). Noting also that ram pressure stripping does not play a major role in the removal of HI from galaxies in, for example, Hickson Compact Groups (Rasmussen et al. 2008), we do not think a ram pressure origin is likely for the Vela Cloud.

To date we have not observed a stellar component or ionized gas associated with the Vela Cloud ( $\$$ 4.1.2). This is not surprising given that the pressure in the Vela Cloud is too low to create new stars $(\S 5.3)$. Additionally older stars could be too diffusely distributed to be detected or the tidal material may only come from the more loosely bound, metal-poor outer disks of the parent galaxies. If the latter is the case, the Vela Cloud would not contain CO either. A caveat is that the Vela Cloud's HI mass is comparable to the total fraction associated with a normal Sc spiral galaxy. Thus if CO were indeed detected, it would suggest that the tidal field of the group helped dredge up gas from the parent galaxies' inner regions. This would be in contrast to an isolated interaction which usually causes this inner gas to flow towards the galactic potential, converting into stars or forming an AGN. 
Clearly a model of this group would be needed to explore the validity of this scenario in which tidal features are stripped away by a group's gravitational potential. In particular, the morphology, kinematic behaviour, and star formation possibilities need to be explored.

\subsection{Fate of the Vela Cloud}

The density enhancements have masses that are comparable to stellar groups on the scale of globular clusters or dwarf galaxies ( $\S 5.1$ ). However, $\S 5.3$ indicates that these enhancements are not Bonnor-Ebert spheres. That is, there is insufficient external pressure to induce the density enhancements into forming stars. Indeed, for the observed velocities, the enhancements could require another order of magnitude of mass in order for an enhancement to collapse. Therefore it is not a surprise that the HII galaxy WPV060 does not appear to be a dwarf galaxy embedded in the Vela Cloud. Also the FWHM velocities in the enhancements are 2-4 times the typical turbulent velocity of ISM $\left(\sim 10 \mathrm{~km} \mathrm{~s}^{-1}\right)$ and it is hard to imagine how the Vela Cloud velocities could be reduced. So unless an event occurs to increase the pressure in each cloud component that hosts an enhancement it is unlikely that these enhancements will be identified in the future with either globular clusters or dwarf galaxies.

A model of the fate of this cloud in the gravitational potential of the group of galaxies would be as interesting as a model of its origin. If there were a possibility of the Vela Cloud collapsing, this would take at least 2 Gyr, ie. the crossing time. This process may be in competition with the time it takes tidal forces to form a structure having this morphology; this timescale is $2-4$ Gyr using the example of the Bekki et al. (2005b) model. If, instead of collapsing, the tidal forces win out then perhaps the enhancements will become stripped of their surrounding medium. If additionally the enhancements become less dense they may be identified with the more typical HVCs that surround our Milky Way, rather than the rare denser CHVCs described in $\S 6.2$, This is of particular interest.

Furthermore, if the stripped enhancements are not accumulated by a specific galaxy they should fall towards the group's centre of mass. Then these "cloudlets" consisting of tidal material, rather than primordial material, may mimic the distribution and motion of primordial building blocks falling into a potential well. The fact that so few enhancement candidates exist in the Vela Cloud has the potential to help discriminate between these 2 scenarios. That is, if too few in-falling objects are observed, compared to the numbers predicted by cosmological simulations, the observed cloudlets are unlikely to be primordial dark matter halos and more likely to be tidal debris from an evolved Vela Cloud-like object. 


\section{Summary}

A rare galaxy-sized intergalactic cloud, the Vela Cloud, which resides in the NGC 3256 Group of galaxies contains, according to our ATCA and Parkes single-dish telescope observations, an HI mass of $3-5 \times 10^{9} \mathcal{M}_{\odot}$. While it is less than the mass in some of the galaxies in this group, this is comparable to the gas content of a normal spiral galaxy.

Since interferometer observations filter out low column densities and specific spatial scales, in ATCA data the cloud appears as 3 separate components containing 4 robust HI density enhancement candidates. We have not discovered a stellar component nor $\mathrm{H} \alpha$ radiation in our preliminary optical investigations. However if parts of the Vela Cloud are within $80 \mathrm{kpc}$ of NGC 3263, then they should be ionized by NGC 3263's UV radiation field. Thus we expect that the Vela Cloud's southeast diagonal component should be observable using the Fabry-Perot staring method.

The position of HII galaxy WPV 060 co-incides with one of the Vela Cloud's prominent density enhancements. However our optical spectra shows that WPV 060's heliocentric systemic velocity differs from the central velocity of the density enhancement by $140 \mathrm{~km} \mathrm{~s}^{-1}$. Since there is no HI in our data at WPV 060's systemic velocity, it is difficult to prove an association. Thus there is no support for a scenario in which the enhancement could be a dwarf galaxy.

It is not surprising that bright stars and stellar systems (such as dwarf galaxies and globular clusters) are not evident in the Vela Cloud. Both its probable origin and the current conditions are not conducive to star formation. A likely origin is suggested by the fact that the galaxies in the group are tidally disturbed and that the Vela Cloud is reminescent of debris in the Virgo cluster. Thus the Vela Cloud could have resulted from the tidal field of the NGC 3256 Group stripping tidal tails from a pair of interacting galaxies. In this case the distribution of stars in the debris could be extremely attenuated or the tidal debris could have been pulled exclusively from the outer disks' HI gas. With respect to current conditions, our exploration indicates that stars will not currently form out of this debris. Although the enhancements within the Vela Cloud contain sufficient mass $\left(\sim 10^{8} \mathcal{M}_{\odot}\right)$ to be considered possible progenitors of globular clusters or dwarf galaxies, their internal pressures $\left(\mathrm{P} / \mathrm{k} \leq 100 \mathrm{~K} \mathrm{~cm}^{-3}\right)$ are less than the $10^{6-8} \mathrm{~K} \mathrm{~cm}^{-3}$ required to instigate the formation of bound stellar structures. Also we used the Bonnor-Ebert formulation to derive a theoretical mass that the enhancements would require in order to form stars. The mass in HI emission is less than this required mass, by factors ranging from 6-44.

The FWHM of the 3 measured density enhancement candidates are similar to those of CHVCs and the column densities are consistent with a some atypical CHVCs. Although 
evidence is building that HVCs orbit close to the Galaxy, any distant CHVCs would have similar diameters to these enhancements if they reside at $\sim 1 \mathrm{Mpc}$ from the Milky Way. If an event does not occur to compress the Vela Cloud, but rather the surrounding diffuse material is stripped away from the enhancements by the overall tidal field the NGC 3256 Group of galaxies, then we speculate that the density enhancements may indeed evolve into the type of object which could be identified with distant CHVC or even more typical HVC.

Discussions with Jason Fiege were exceedingly useful and with Dean McLauglin were inspiring. We thank Marija Vlajic for her contribution to Fig. 11, J. E. acknowledges the support from Australia Telescope National Facility in the form of a Distinguished Visitor Award and from the Canadian National Science and Engineering Research Council.

\section{REFERENCES}

Bekki, K., Koribalski, B. S., \& Kilborn, V. A. 2005a, MNRAS, 323, L21

Bekki, K., Koribalski, B. S., Ryder, S. D., \& Couch, W. J. 2005b, MNRAS, 357, L21

Binney, J., \& Merrifield, M. 1998, Galactic astronomy Princeton, NJ : Princeton University Press, 1998.

Bland-Hawthorn, J., \& Maloney, P. R. 1999, ApJ, 510, L33

Bland-Hawthorn, J., \& Maloney, P. R. 2002, in ASP Conf. Ser. 254: Extragalactic Gas at Low Redshift, 267

Bland-Hawthorn, J., Taylor, K., Veilleux, S., \& Shopbell, P. L. 1994, ApJ, 437, L95

Bland-Hawthorn, J., Veilleux, S., Cecil, G. N., Putman, M. E., Gibson, B. K., \& Maloney, P. R. 1998, MNRAS, 299, 611

Blitz, L., Spergel, D. N., Teuben, P. J., Hartmann, D., \& Burton, W. B. 1999, ApJ, 514, 818

Bonnor, W. B. 1956, MNRAS, 116, 351

Bothun, G. D., \& Sullivan, W. T. 1980, ApJ, 242, 903

Dickey, J. M. \& Lockman, F. J. 1990, ARA\&A, 28, 215

Côté, S., Carignan, C., \& Freeman, K. C. 2000, AJ, 120, 3027

de Heij, V., Braun, R., \& Burton, W. B. 2002a, A\&A, 392, 417 
-. 2002b, A\&A, 391, 159

Dubinski, J. 1998, ApJ, 502, 141

Duc, P.-A., Bournaud, F., \& Masset, F. 2004, A\&A, 427, 803

, Duc, P.-A. and Bournaud, F. 2008, ApJ, 673, 787

Elmegreen, B. G., \& Efremov, Y. N. 1997, ApJ, 480, 235

English, J. 1994, PhD thesis, Australian National University

English, J., \& Freeman, K. C. 2003, AJ, 125, 1124

English, J., Koribalski, B. S., \& Freeman, K. C. 2004, in IAU Symposium 217, 41

English, J., Norris, R. P., Freeman, K. C., \& Booth, R. S. 2003, AJ, 125, 1134

Fouque, P., Gourgoulhon, E., Chamaraux, P., \& Paturel, G. 1992, A\&AS, 93, 211

Fraternali, F. and Binney, J. J. 2006, MNRAS, 366, 449

Fraternali, F. and Binney, J. J. 2008, MNRAS, 386, 935

Fraternali, F., van Moorsel, G., Sancisi, R., \& Oosterloo, T. 2002, AJ, 123, 3124

Ganguly, R., Sembach, K. R., Tripp, T. M., \& Savage, B. D. 2005, ApJS, 157, 251

Garcia, A. M. 1993, A\&AS, 100, 47

Gooch, R. 1996, in ASP Conf. Ser. 101: Astronomical Data Analysis Software and Systems $\mathrm{V}, 80-+$

Hambly,N. C. ,MacGillivray, H. T., Read, M. A., Tritton, S. B., Thomson, E. B., Kelly, B. D., Morgan, D. H., Smith, R. E., Driver, S. P., Williamson, J., Parker, Q. A., Hawkins, M. R. S., Williams, P. M., Lawrence, A. 2001, MNRAS, 326, 1279

Haynes, M. P. and Giovanelli, R. and Kent, B. R. 2007, ApJ, 665, L19

Hibbard, J. E., van der Hulst, J. M., Barnes, J. E., \& Rich, R. M. 2001, AJ, 122, 2969

Hibbard, J. E.,van Gorkom, J. H., Rupen, M. P. \& Schiminovich, D. 2001, ASP Conf. Ser., 240,657

Irwin, J. A., \& Chaves, T. 2003, ApJ, 585, 268 
Kalberla, P. M. W., \& Haud, U. 2006, A\&A, 455, 481

Kent, B. R. 2008, IAU Symposium 244, eds. Davies, J. and Disney, M., 93

Klypin, A., Kravtsov, A. V., Valenzuela, O., \& Prada, F. 1999, ApJ, 522, 82

Koribalski, B. 2004, in IAU Symposium 217, 34

Koribalski, B., \& Dickey, J. M. 2004, MNRAS, 348, 1255

Koribalski, B., Gordon, S., \& Jones, K. 2003, MNRAS, 339, 1203

Lípari, S., Díaz, R., Taniguchi, Y., Terlevich, R., Dottori, H., \& Carranza, G. 2000, AJ, 120, 645

Mihos, J. C. 2004a, in Clusters of Galaxies: Probes of Cosmological Structure and Galaxy Evolution, 278

Mihos, J. C. 2004b, in IAU Symposium, 390

Miller, E. D., \& Bregman, J. N. 2005, ASPC, 331, 261

Mould, J. R., Han, M. S., Roth, J., Staveley-Smith, L., Schommer, R. A., Bothun, G. D., Hall, P. J., Huchra, J. P., Walsh, W., \& Wright, A. E. 1991, ApJ, 383, 467

Minchin, R., Davies, J., Disney, M., Grossi, M., Sabatini, S., Boyce, P., Garcia, D., Impey, C., Jordan, C., Lang, R., Marble, A., Roberts, S. and \& van Driel, W. 2007, ApJ, 670,1056

Norman, C. A., \& Ikeuchi, S. 1989, ApJ, 345, 372

Oosterloo, T., \& van Gorkom, J. 2005, A\&A, 437, L19

Oosterloo, T., Fraternali, F., Sancisi, R. 2007, AJ, 134,1019

Ott, J., Walter, F., \& Brinks, E. 2005, MNRAS, 358, 1453

Patel, K., \& Wilson, C. D. 1995, ApJ, 451, 607

Pisano, D. J., Barnes, D. G., Gibson, B. K., Staveley-Smith, L., Freeman, K. C., \& Kilborn, V. A. 2004, ApJ, 610, L17

Pisano, D. J., Barnes, D. G., Gibson, B. K., Staveley-Smith, L., Freeman, K. C., \& Kilborn, V. A. 2007, ApJ, 662, 959 
Peek, J. E. G., Putman, M. E., Sommer-Larsen, J. 2006, ApJ, 674, 227

Putman, M. E., Bland-Hawthorn, J., Veilleux, S., Gibson, B. K., Freeman, K. C., \& Maloney, P. R. 2003a, ApJ, 597, 948

Putman, M. E., Bureau, M., Mould, J. R., Staveley-Smith, L., \& Freeman, K. C. 1998, AJ, 115,2345

Putman, M. E., de Heij, V., Staveley-Smith, L., Braun, R., Freeman, K. C., Gibson, B. K., Burton, W. B., Barnes, D. G., Banks, G. D., Bhathal, R., de Blok, W. J. G., Boyce, P. J., Disney, M. J., Drinkwater, M. J., Ekers, R. D., Henning, P. A., Jerjen, H., Kilborn, V. A., Knezek, P. M., Koribalski, B., Malin, D. F., Marquarding, M., Minchin, R. F., Mould, J. R., Oosterloo, T., Price, R. M., Ryder, S. D., Sadler, E. M., Stewart, I., Stootman, F., Webster, R. L., \& Wright, A. E. 2002, AJ, 123, 873

Putman, M. E., Staveley-Smith, L., Freeman, K. C., Gibson, B. K., \& Barnes, D. G. 2003b, ApJ, 586, 170

Quinn, P. J., \& Goodman, J. 1986, ApJ, 309, 472

Rand, R. J. Benjamin, R. A. 2008, ApJ, 676, 991

Rasmussen, J., Ponman, T. J., Verdes-Montenegro, L., Yun, M. S. \& Borthakur, S. 2008, MNRAS, 388, 1245

Rector, T., Levay, Z., Frattare, L. M., English, J., \& Pu'uohau-Pummill, K. 2007, AJ, 133, 598

Ryder, S. D., Koribalski, B., Staveley-Smith, L., Kilborn, V. A., Malin, D. F., Banks, G. D., Barnes, D. G., Bhatal, R., de Blok, W. J. G., Boyce, P. J., Disney, M. J., Drinkwater, M. J., Ekers, R. D., Freeman, K. C., Gibson, B. K., Henning, P. A., Jerjen, H., Knezek, P. M., Marquarding, M., Minchin, R. F., Mould, J. R., Oosterloo, T., Price, R. M., Putman, M. E., Sadler, E. M., Stewart, I., Stootman, F., Webster, R. L., \& Wright, A. E. 2001, ApJ, 555, 232

Ryder, S. D. \& Koribalski, B. S. 2004, in IAU Symposium 217, 44

Schneider, S. E., Helou, G., Salpeter, E. E., \& Terzian, Y. 1983, ApJ, 273, L1

Siegel, M. H., Majewski, S. R.Gallart, C., Sohn, S. T., Kunkel, W. E., \& Braun, R. 2005, ApJ, 623, 181

Simon, J. D. Blitz, L., Cole, A. A., Weinberg, M. D. \& Cohen, M. 2006, ApJ,640, 270 
Simon, J. D. \& Blitz, L. 2003, ApJ, 574, 762

Taylor, C., Brinks, E., \& Skillman, E. D. 1993, AJ, 105, 128

Taylor, C. L., Brinks, E., Grashuis, R. M., \& Skillman, E. D. 1995, ApJS, 99, 427

Taylor, C. L., Brinks, E., Grashuis, R. M., \& Skillman, E. D. 1996, ApJS, 102, 189

Thilker, D. A., Braun, R., Walterbos, R. A. M., Corbelli, E., Lockman, F. J., Murphy, E., \& Maddalena, R. 2004, ApJ, 601, L39

van Woerden, H., Schwarz, U. J., Peletier, R. F., Wakker, B. P., \& Kalberla, P. M. W. 1999, Nature, 400, 138

Veilleux, S., Shopbell, P. L., Rupke, D. S., Bland-Hawthorn, J., \& Cecil, G. 2003, AJ, 126, 2185

Wakker, B. P., \& Schwarz, U. J. 1991, A\&A, 250, 484

Wakker, B. P., \& van Woerden, H. 1997, ARA\&A, 35, 217

Wakker, B. P., York, D. G., Wilhelm, R., Barentine, J. C., Richter, P., Beers, T. C., Ivezi, ., \& Howk, J. C. 2008, ApJ, 672, 298

Walter, F., Skillman, E. D., \& Brinks, E. 2005, ApJ, 627, L105

Westmeier, T., Braun, R., \& Thilker, D. 2005, A\&A, 436, 101

Westmeier, T., Braun, R., \& Thilker, D. 2007, NewAR, 51, 108

Westmeier, T. \& Koribalski, B. S. 2008, MNRAS, 388, L29

Weymann, R. J. Vogel, S. N. Veilleux, S. \& Epps, H. W. 2001, ApJ, 561, 559

Willman, B., Dalcanton, J., Ivezić, Ž., Schneider, D. P., \& York, D. G. 2002, AJ, 124, 2600

Winkler, H., Stirpe, G. M., \& Sekiguchi, K. 1992, A\&AS, 94, 103

Zwaan, M. A. 2001, MNRAS, 325, 1142 


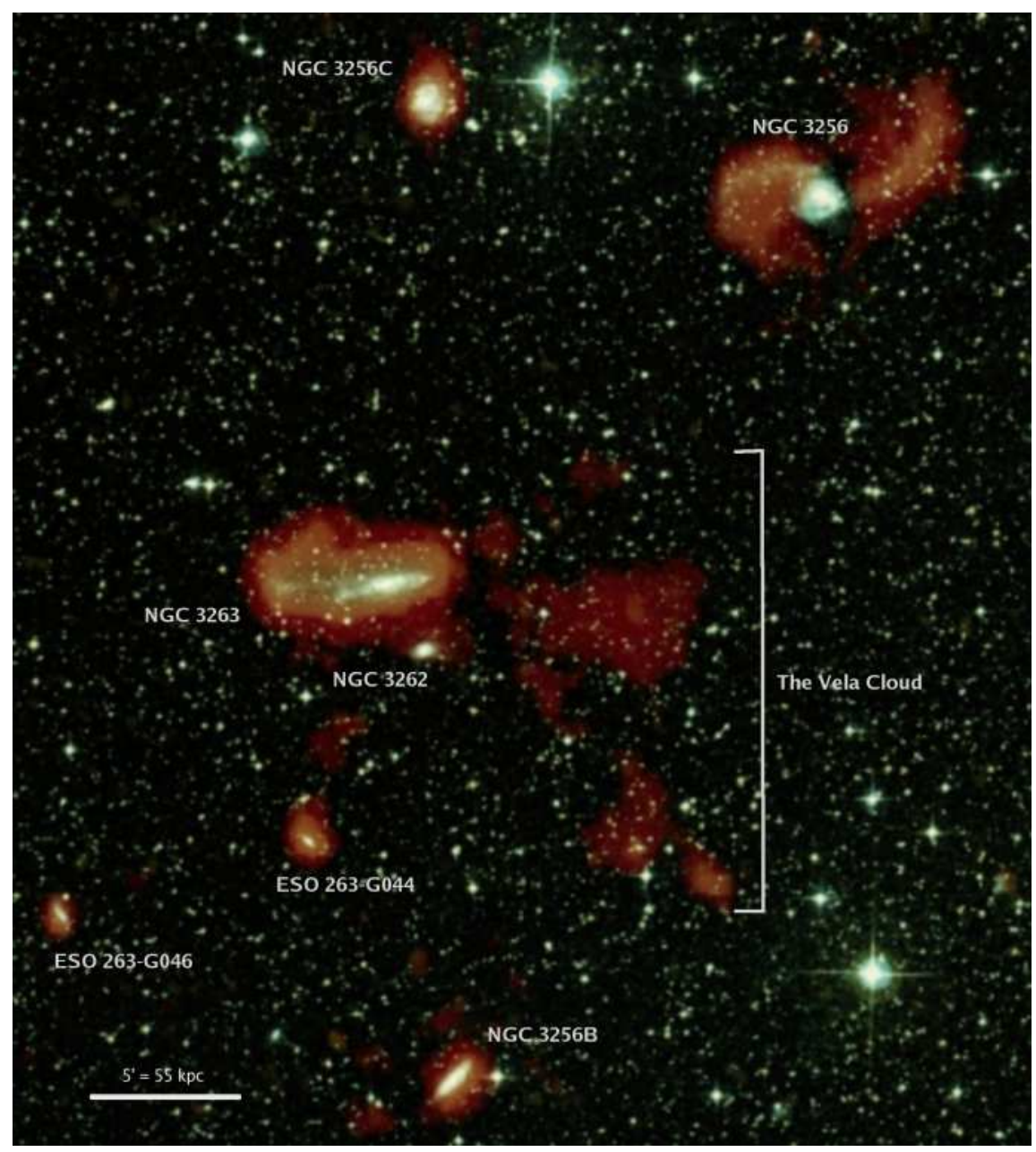

Fig. 1.- HI distribution in the NGC 3256 Group of galaxies. The HI distribution shown is the combination of 2 different ATCA moment maps, both of which are integrated from 2621 to $3337 \mathrm{~km} \mathrm{~s}^{-1}$. One map was generated from a cube with a naturally weighted synthesized beam of $83^{\prime \prime} \times 67^{\prime \prime}$ while the other map is from a cube with an approximately uniformly weighted beam with dimensions $58^{\prime \prime} \times 67^{\prime \prime}$. The initial greyscale intensity stretches were logarithmic in order to emphasize fainter emission. The lower resolution map was assigned a darker red than the higher resolution map. The maps were then combined using the screen algorithm in "The GIMP" image editing package; The GIMP is written by Peter Mattis and Spencer Kimball, and released under the GNU General Public License. We used image editing techniques described in Rector et al. (2007). The HI emission maps have been overlaid on colourized blue and red Digitized Sky Survey images. North is up and east is to the left. In these ATCA data, the Vela Cloud appears as 3 components to the west of NGC 3263, which is the edge-on galaxy near the center of the image. The cloud is plotted in more detail, with coordinates, in Fig. 5 . 

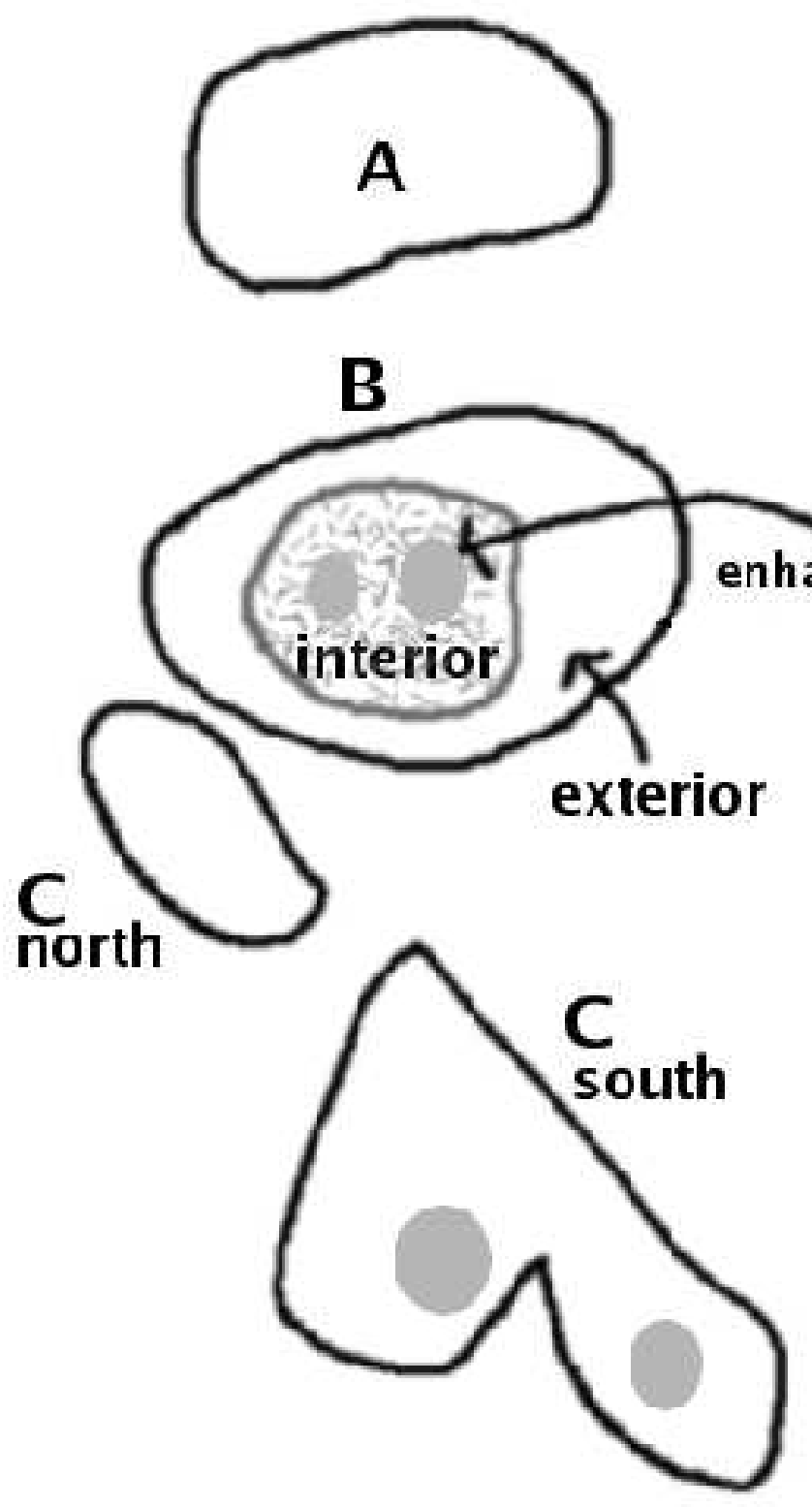

Fig. 2.- Schematic of the components of the Vela Cloud as derived from the HI cube (Table 1). Diffuse cloud components are labelled A, B and C and are apparent in Fig. 5. Note that B appears to have an exterior diffuse envelope surrounding a more structured interior component (enclosed by the grey contour); the pressures of the envelope and the structured interior are compared in $\S 5.2 .2$. The structured interior in turn contains 2 enhancements. $\mathrm{C}$ has a northern diffuse section and a southern section which contains 2 enhancements. The models of the enhancements, described in $\S 3.2$, are represented here using grey ellipses. 


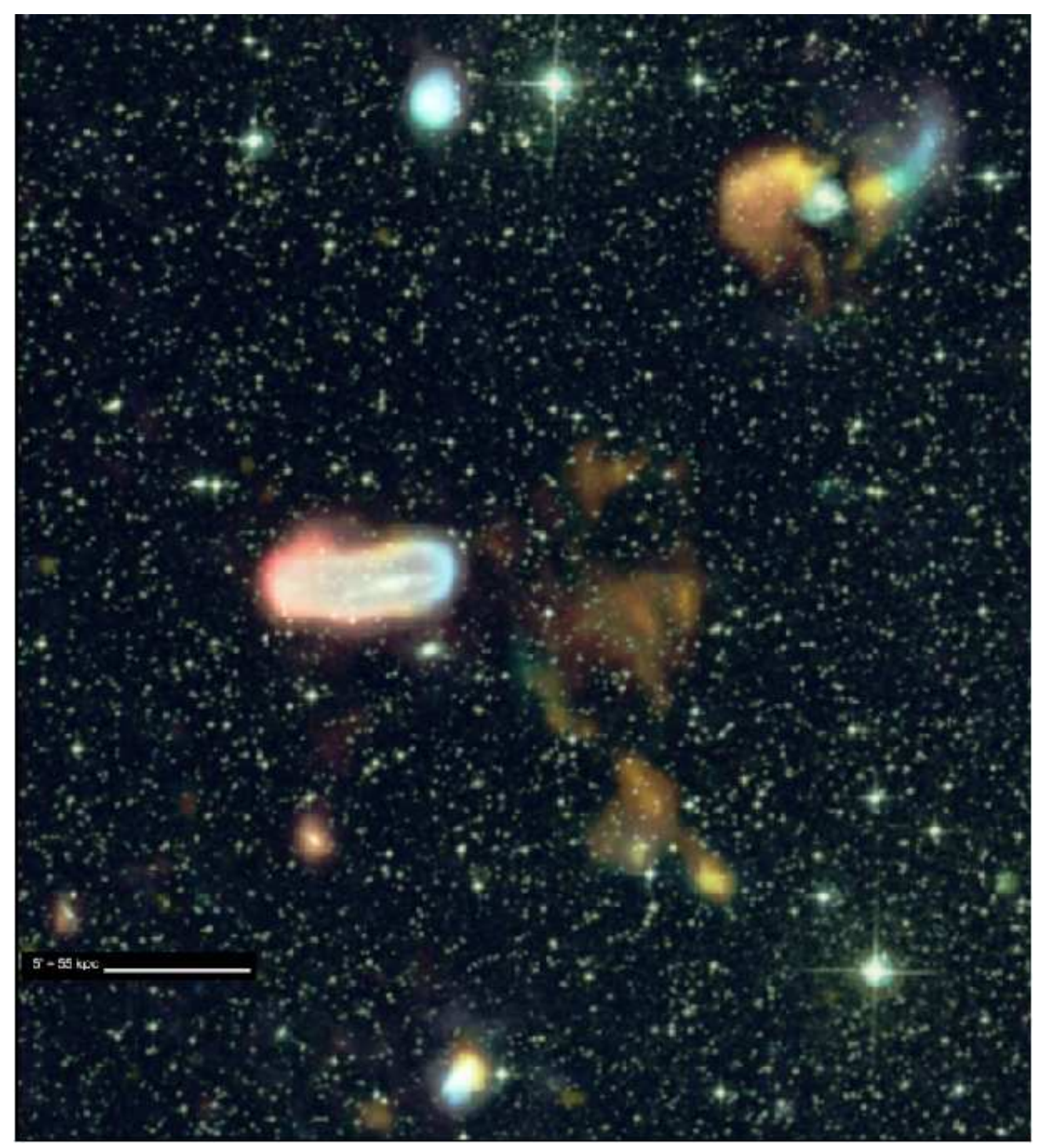

Fig. 3.- HI velocity field in the NGC 3256 Group of galaxies. The HI cubes were the same as those used for creating the moment maps in Fig.1. For each of these cubes individual integrated intensity maps were created for 8 separate velocity ranges between 2621 to 3337 $\mathrm{km} \mathrm{s}^{-1}$. Initially each map was displayed with a linear intensity stretch and adjusted in order to emphasize the brighter features in the cloud, generating a greyscale image. Each greyscale image was subsequently colour coded such that blue represents blueshifted $21 \mathrm{~cm}$ emission and red represents redshifted emission. The radio emission maps from both cubes have been overlaid, using the screen algorithm (Rector et al. 2007), on colourized Digitized Sky Survey images from the blue and the red wavelength regimes. (Reprinted, with permission, from the cover of I.A.U. Sym 2172004 "Recycling Intergalactic \& Interstellar Matter", editors Pierre-Alain Duc, Jonathan Braine, and Elias Brinks, A.S.P. 217.) 


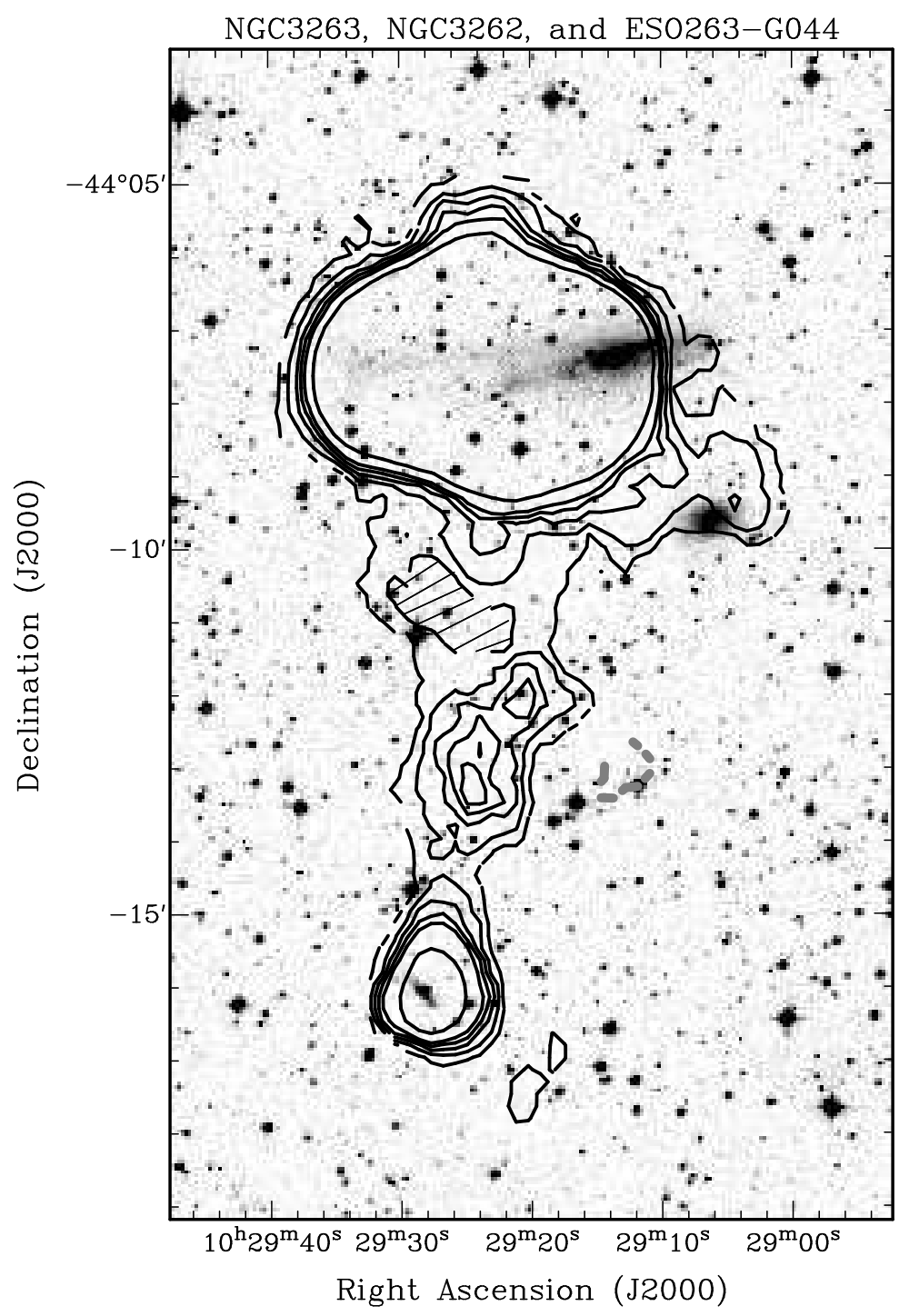

Fig. 4.- The possible HI bridge between NGC 3263 and ESO 263-G044. Contours from an HI intensity map, integrated from 3025 to $3171 \mathrm{~km} \mathrm{~s}^{-1}$, overlay a greyscale Digitized Sky Survey image. (Values between $\pm 3 \mathrm{mJy}$ beam $^{-1}$ (that is, $\pm 3 \sigma$ ) were clipped from the zeroth moment map.) West (right) of NGC 3263 lies NGC 3262 while ESO 263-G044 lies to the south (bottom). The HI intensity contours delineating the bridge and outlining the galaxies are $60120180240300480 \mathrm{mJy}$ beam $^{-1} \times \mathrm{km} \mathrm{s}^{-1}$. The dashed grey contour (to the right of the bridge) is $-30 \mathrm{mJy}$ beam $^{-1} \times \mathrm{km} \mathrm{s}^{-1}$. No HI emission resides in the lightly hashed area. See $\S 6.1$ for a more detailed description. 


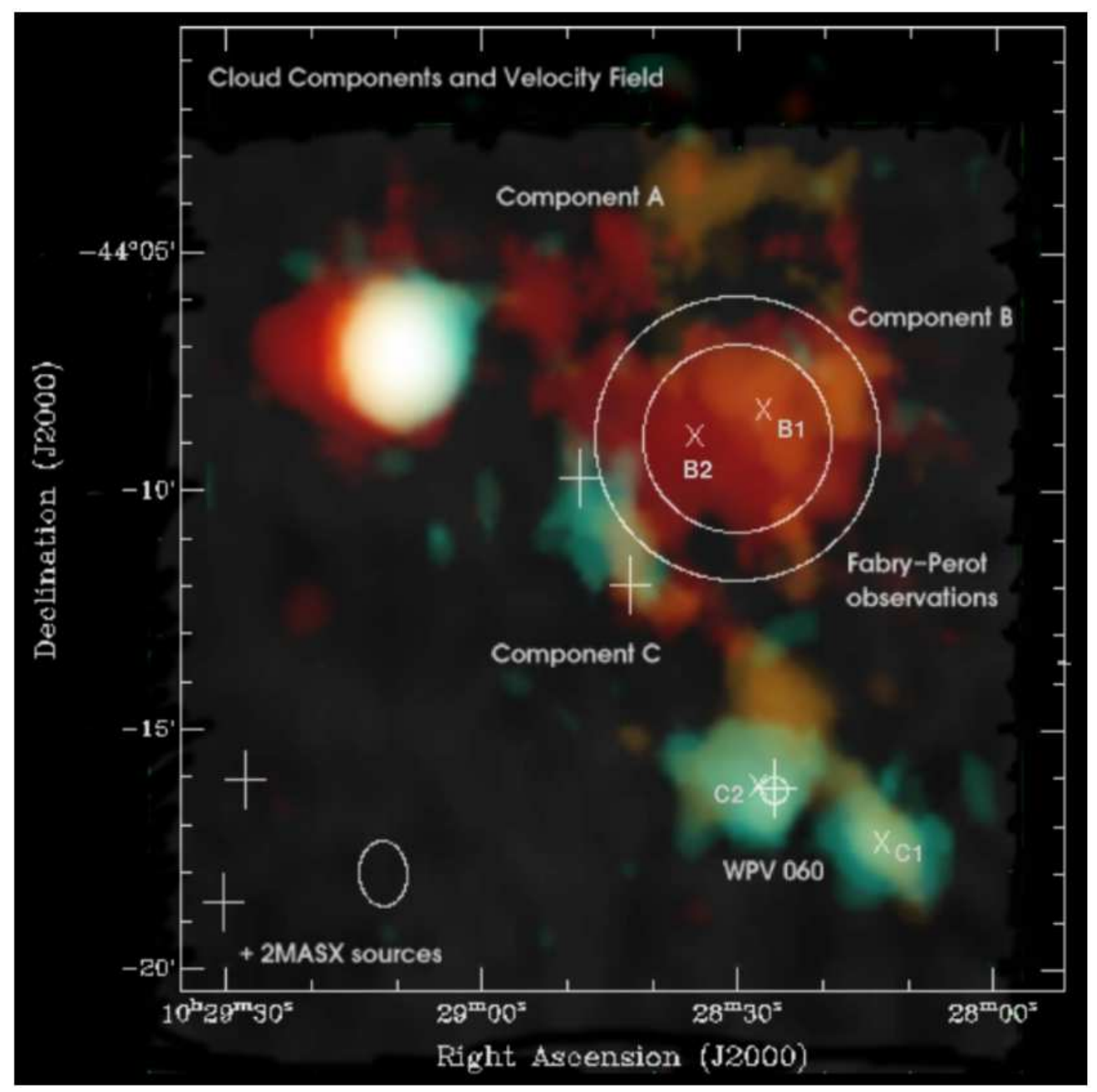

Fig. 5.- Features in the Mean HI Velocity Field of the Vela Cloud. Three integrated intensity maps were made from a lower velocity resolution $\left(10 \mathrm{~km} \mathrm{~s}^{-1}\right)$ HI cube than the cube presented in Table 1. The colour turquoise was assigned to the map covering velocities from roughly 2773 through $2830 \mathrm{~km} \mathrm{~s}^{-1}$; gold to $2831-2858 \mathrm{~km} \mathrm{~s}^{-1}$; and dark red to 2859 $2940 \mathrm{~km} \mathrm{~s}^{-1}$. The bright white feature to the east is part of NGC 3263. Component A arcs horizontally from NGC 3263 to the west; component B is the elliptically shaped emission cloud below $\mathrm{A}$; and component $\mathrm{C}$ runs on a diagonal from the east edge of $\mathrm{B}$ to the southwest. These 3 morphological components are described in more detail in $\S 4.1$ and sketched in Fig. 2, X-shaped crosses, with letter designations, mark positions of substructures found in the higher velocity resolution spectra of the cube listed in Table 1 and described in $\S$ 4.2. Plus-shaped crosses mark 2MASS infra-red sources. WPV060, designated by a circle, is a starburst galaxy also discussed in $\S 4.2$. For scale, ten seconds of right ascension, using a declination of $-44^{\circ} 09^{\prime}$, is about $1.8^{\prime}$ and, at a distance of $37.6 \mathrm{Mpc}, 1^{\prime}$ equals $11 \mathrm{kpc}$. 


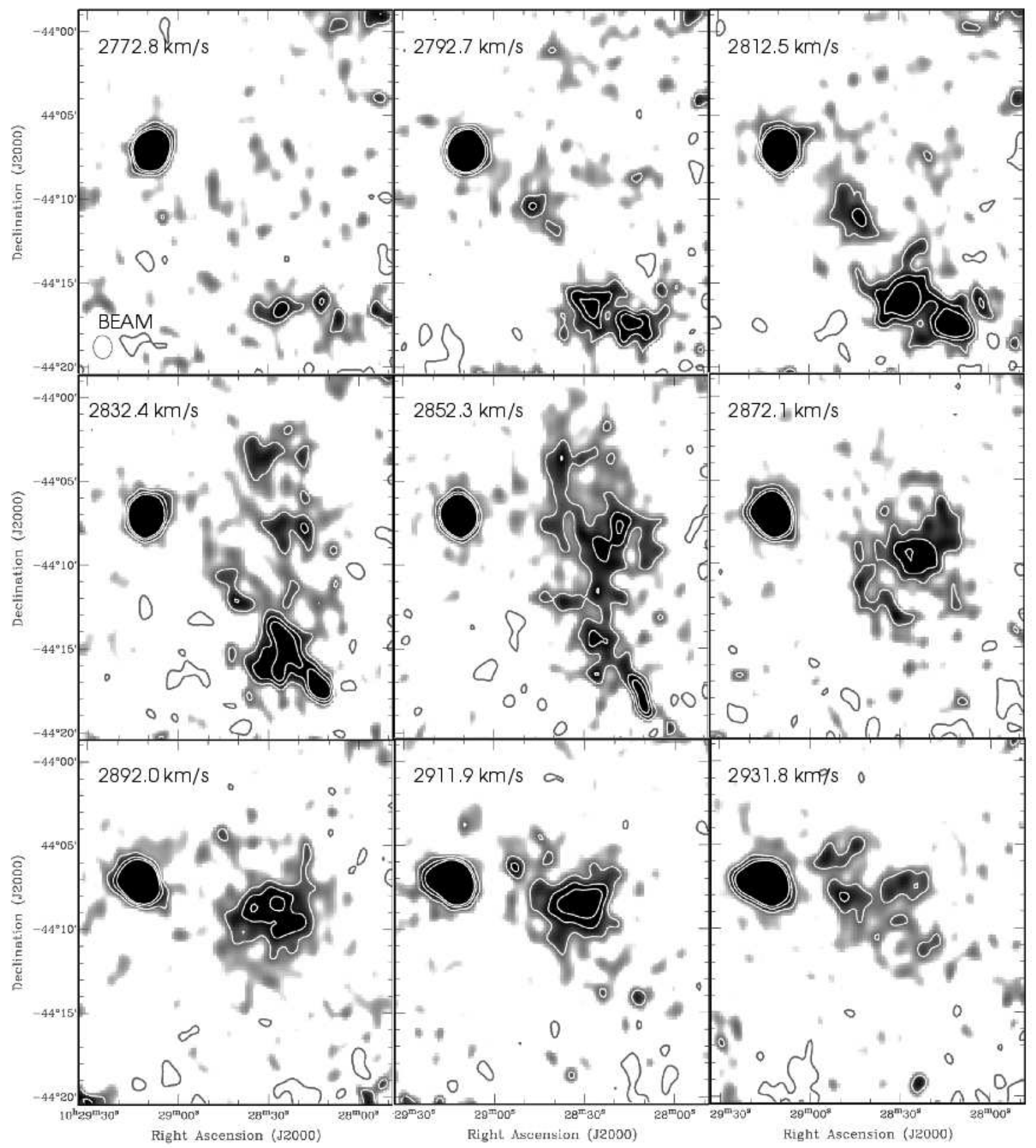

Fig. 6. - HI channel maps of the Vela Cloud. Each plane consists of three combined channels from the HI cube (Table 1) and has a velocity width of $\sim 20 \mathrm{~km} \mathrm{~s}^{-1}$. The greyscale ranges from $1 \mathrm{mJy}_{\text {beam }^{-1}}(\sim 1 \sigma)$ to $5 \mathrm{mJy}_{\text {beam }}{ }^{-1}$. The dark contours are $-3 \mathrm{mJy}_{\text {beam }}{ }^{-1}$ while the white contours are 3,5 , and $7 \mathrm{mJy}_{\text {beam }}{ }^{-1}$. The enhancements in component $\mathrm{C}$ are clear at $2792.7 \mathrm{~km} \mathrm{~s}^{-1}$ while the enhancements in component B are evident at $2892 \mathrm{~km} \mathrm{~s}^{-1}$. Note that all 3 components appear associated at $2852.3 \mathrm{~km} \mathrm{~s}^{-1}$. The strong circular feature to the east (centered near $10^{\mathrm{h}} 29^{\mathrm{m}} 10^{\mathrm{s}}$, $-44^{\circ} 07^{\prime}$ ) that appears in every panel is part of NGC 3263 . 

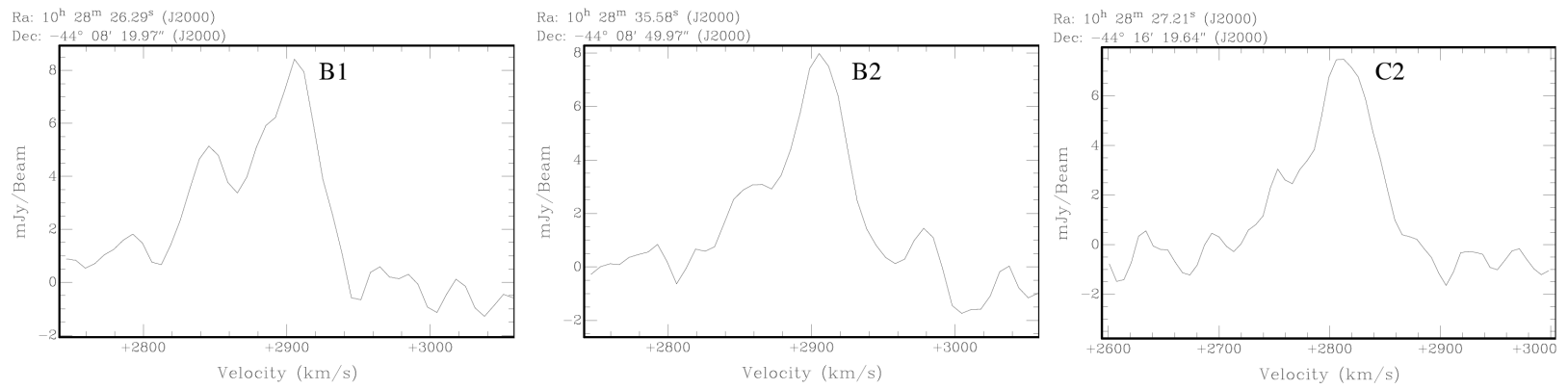

Fig. 7.- Velocity profiles of the enhancements. These profiles, while covering the spatial region of the enhancements, include emission in the velocity range of the host component (§3.2,4.2). Each labelled peak is associated with an enhancement's velocity range assessed visually within the data cube.

Editor: Please rotate this figure to landscape mode so that the lines show well. Also ensure that it is on a separate page. 

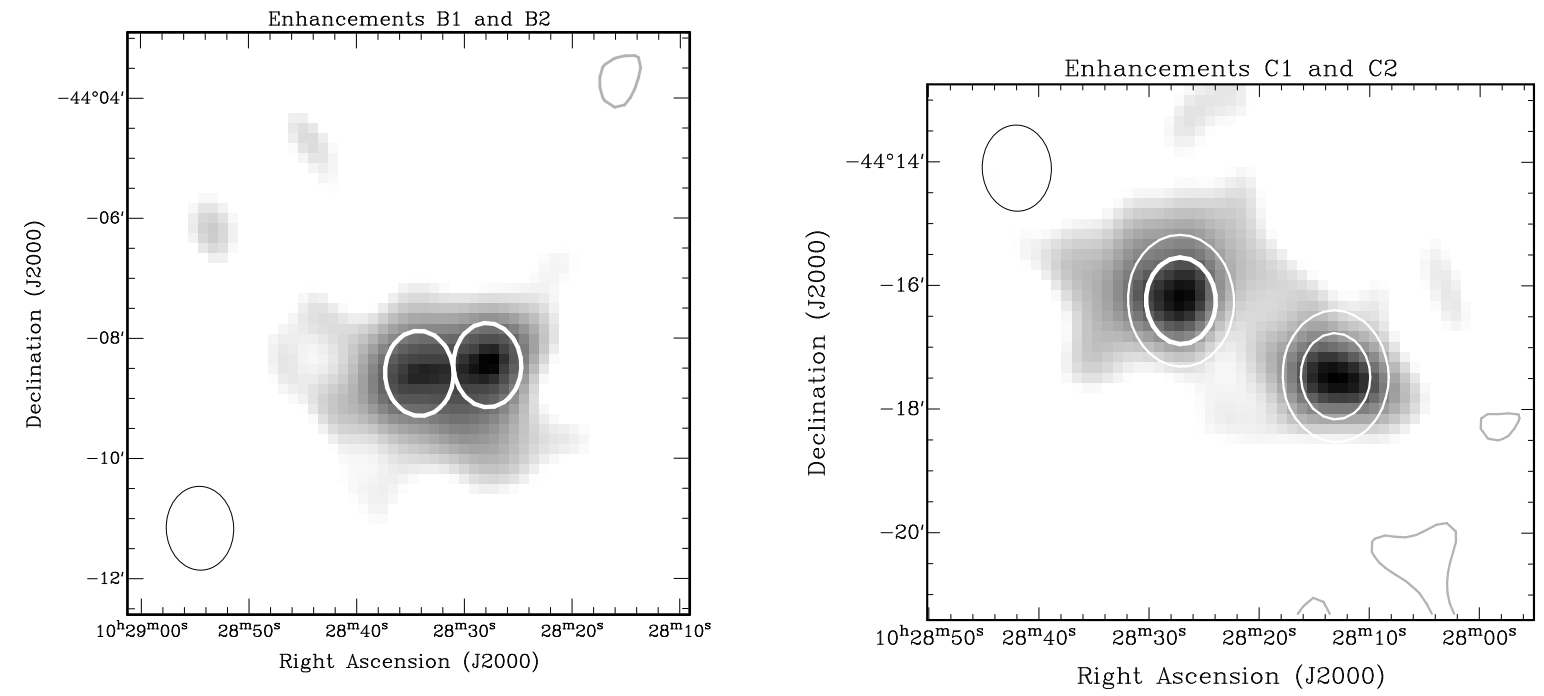

Fig. 8.- Integrated HI intensity maps of the enhancements. The candidate enhancements in each cloud appear in integrated intensity maps constructed from cubes in which the flux level of the surrounding diffuse cloud has been subtracted ( $\$ 3.2$ ). For the left-hand image containing B1 and B2, the non-linear greyscale displays values in the range of 0.3 to 200.1 mJy beam ${ }^{-1} \times \mathrm{km} \mathrm{s}^{-1}$. For the greyscale image containing $\mathrm{C} 1$ and $\mathrm{C} 2$ the values are 0.6 to $246.7 \mathrm{mJy}_{\text {beam }}^{-1} \times \mathrm{km} \mathrm{s}^{-1}$. For comparison of noise features with the shapes of the enhancements, we show grey contours which trace $-190 \mathrm{mJy}^{\mathrm{beam}}{ }^{-1} \times \mathrm{km} \mathrm{s}^{-1}$. The white contours outline the $50 \%$ contour of the Gaussian model fit used to measure the flux in each enhancement in Cloud B and the 20\% and 50\% levels in Cloud C; see $\S$ 3.2. The black ellipse, in the lower left corner, outlines the FWHM of the synthesized beam. 

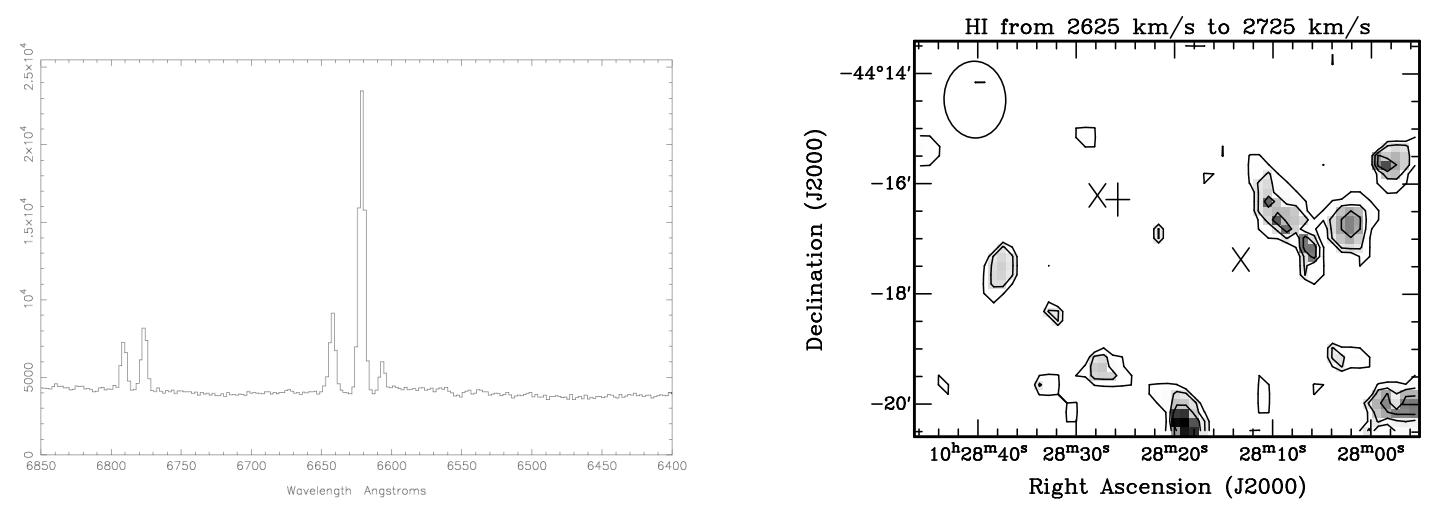

Fig. 9.- Galaxy WPV060. See $\S 4.2$ for discussion. Details about the optical observations for the spectrum on the left are described in $\S 2.2$. See $\S 4.2$ for the discussion associated with this figure. The redshifted optical emission lines give a heliocentric systemic velocity for WPV060 of $2675 \pm 6 \mathrm{~km} \mathrm{~s}^{-1}$. The map on the right integrates the HI intensities in the velocity range between 2625 and $2725 \mathrm{~km} \mathrm{~s}^{-1}$, which is centred on the peak optical velocity of WPV060. This demonstrates that, at the spatial scale of this HI cube (Table 1), there is no emission associated with this HII galaxy; the features in the plot are noise. The beam is represented by the ellipse in the upper left corner. Only intensities above $3 \sigma$ where used; the contours are $4(=3 \sigma), 8$, and $12 \mathrm{mJy}_{\text {beam }}{ }^{-1}$. The position of $\mathrm{C} 2$ is marked by the $\times$ on the left and $\mathrm{C} 1$ is the $\times$ on the right. The + marking the position of WPV060 is larger than the galaxy's diameter of $\sim 7^{\prime \prime}$.

Editor: Please rotate this figure to landscape mode so that the lines show well. 


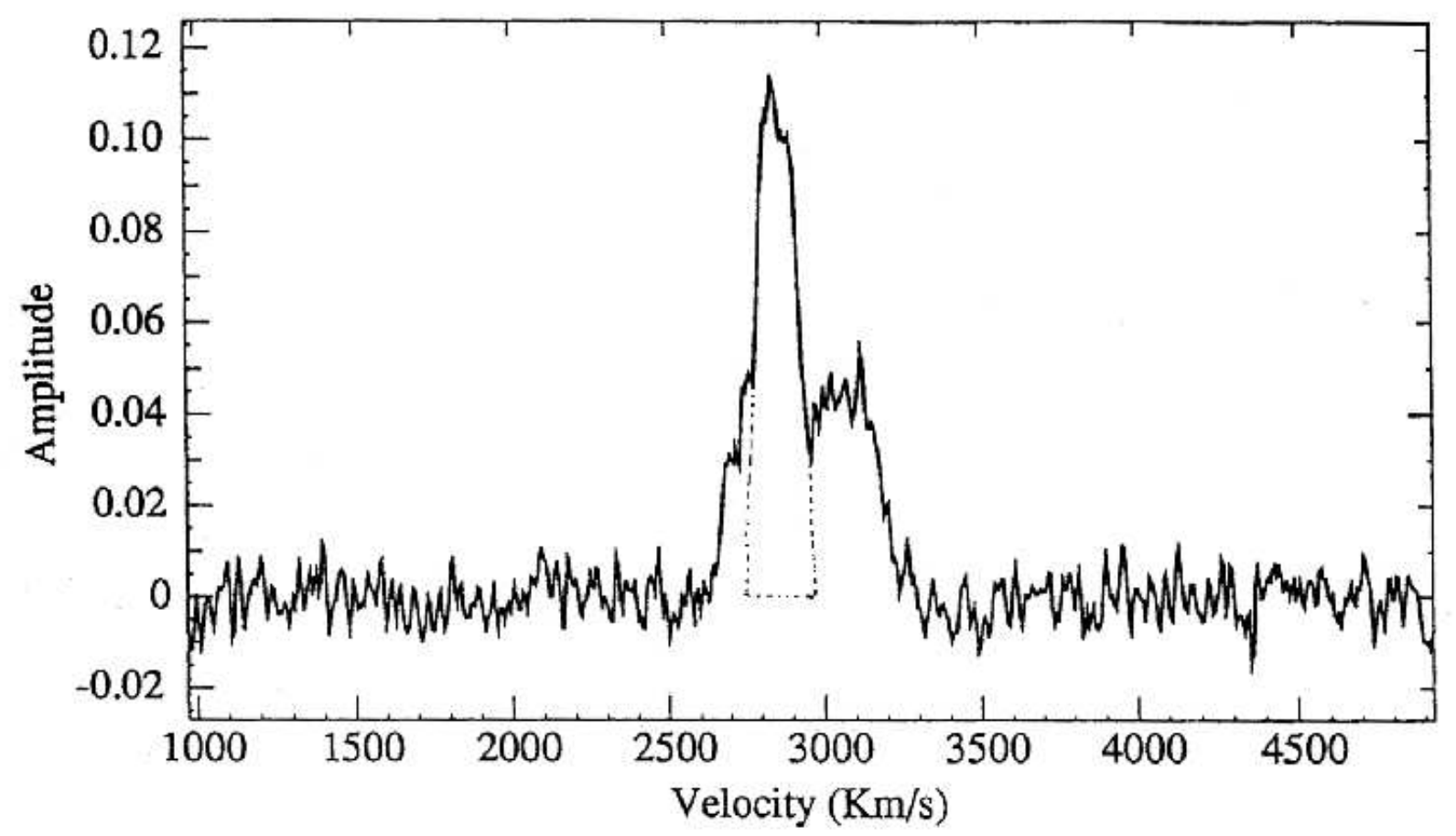

Fig. 10.- Parkes Single-Dish Telescope Profile. Amplitude is in units of Jy. The on-target position is $\alpha=10^{\mathrm{h}} 28^{\mathrm{m}} 27^{\mathrm{s}} .7, \delta=-44^{\circ} 09^{\prime} 26^{\prime \prime}(\mathrm{J} 2000)$. The area under the peak, enclosed in a dashed line, is believed to be associated with the Vela Cloud rather than NGC 3263; more detail is given in $\S$ and English 1994. This area is used to determine the mass of the Vela Cloud in $\S$, 


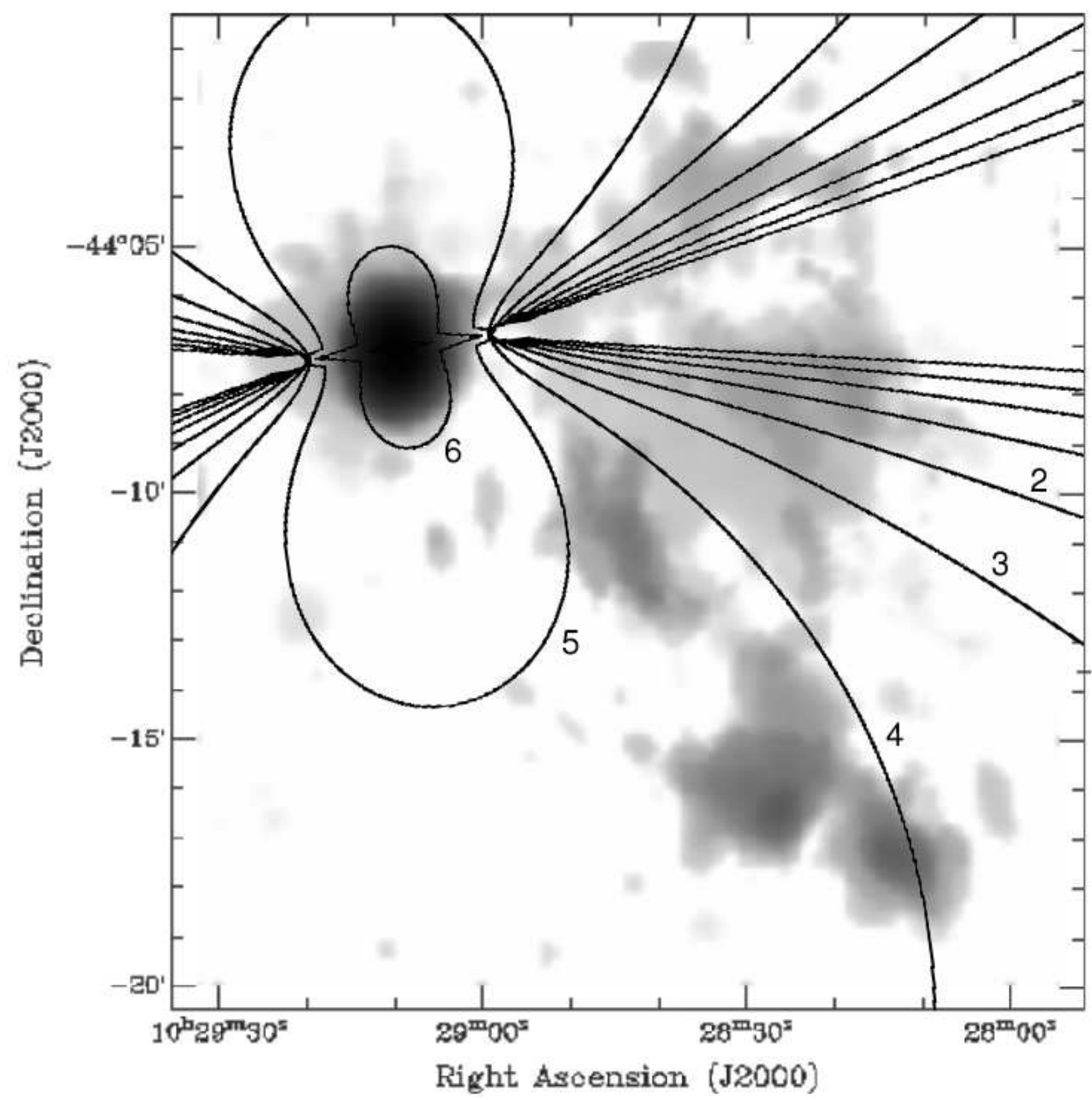

Fig. 11.- The distribution of the ionizing radiation above and below the plane of NGC 3263 (position angle $=10$ degrees) superimposed on a $\mathrm{B} \& \mathrm{~W}$ rendition of the HI velocity data in Fig. 5. The model is overlaid on the optical centre of NGC 3263 and the lines show a crosssection through the radiation field. The contours represent lines of equal ionizing flux. The units are $\log \left(\right.$ photons $\left.\mathrm{cm}^{-2} \mathrm{~s}^{-1}\right)$. See $\S 5.4$. 
Table 1. Australia Telescope Compact Array HI Data Cube: Characteristics of the position-velocity cube created from 21-cm spectral line data. Subcubes with smaller velocity ranges were produced for the various analyses described in $\S 3$.

\begin{tabular}{lc}
\hline \hline \multicolumn{1}{c}{ Parameter } & HI Cube \\
\hline Synthesized Beam (arcsec) & $84 \times 67$ \\
Beam Position Angle & $2.5^{\circ}$ \\
Channel Width (km s & 6.6 \\
Weighting & natural \\
Measured r.m.s. ${ }^{\text {a }}\left(\mathrm{mJy} \mathrm{beam}^{-1}\right)$ & 1.3 \\
Total Velocity Range $\left(\mathrm{km} \mathrm{s}^{-1}\right)$ & 2330 to 3416 \\
\hline
\end{tabular}

aThe r.m.s. was determined before correction for the primary beam.

Table 2. AAO Fabry-Perot Integration Times. These observations attempt to detect $\mathrm{H} \alpha$ in Cloud B. See $\S 2.2$ for details such as the coordinates of the field centre, central wavelength and passband width.

\begin{tabular}{cccc}
\hline \hline Target & Exposure Time (sec) & Airmass & Description \\
\hline The Vela Cloud & 1200 & 1.3 & target field \\
The Vela Cloud & 1200 & 1.1 & target field \\
The Vela Cloud & 1200 & 1.1 & target field \\
The Vela Cloud & 1200 & 1.0 & target field \\
EG 193 & $120 \times 6$ & 1.5 & photometric standard \\
\hline
\end{tabular}


Table 3. Measured and derived characteristics of the 3 diffuse components of the Vela Cloud. Measurements, analysis, and assessment of uncertainties of the observables are in §3.1. Derivations of mass, pressure and uncertainties are described in $\$ 5.2 .1$.

\begin{tabular}{|c|c|c|c|c|c|}
\hline & \multicolumn{5}{|c|}{ Cloud Component } \\
\hline & \multirow[t]{2}{*}{$\mathrm{A}$} & \multicolumn{3}{|c|}{$\mathrm{B}$} & \multirow[t]{2}{*}{$\mathrm{C}$} \\
\hline & & Whole & Interior $^{\mathrm{a}}$ & Exterior & \\
\hline $\begin{array}{l}\text { Heliocentric Systemic } \\
\text { Velocities }\left(\mathrm{km} \mathrm{s}^{-1}\right)\end{array}$ & $2842 \pm 4$ & $2885 \pm 6$ & $2905 \pm 6$ & $\cdots$ & $2813 \pm>7$ \\
\hline FWHM $\left(\mathrm{km} \mathrm{s}^{-1}\right)^{\mathrm{b}}$ & 41 & 97 & 51 & 97 & $\ldots$ \\
\hline $\begin{array}{l}\text { Velocity Range } \\
\left(\mathrm{km} \mathrm{s}^{-1}\right)\end{array}$ & $2832-2852$ & 2832-2938 & 2872-2932 & $\cdots$ & $2786-2839$ \\
\hline Length $(\operatorname{arcsec})^{\mathrm{c}}$ & $220 \pm 26$ & $368 \pm 18$ & $175 \pm 5$ & $\ldots$ & $760 \pm 10$ \\
\hline Width (arcsec) & $140 \pm 14$ & $300 \pm 20$ & $130 \pm 9$ & $\ldots$ & $260 \pm 10$ \\
\hline $\begin{array}{l}\text { Flux Density } \\
\left(\mathrm{Jy} \mathrm{km} \mathrm{s}^{-1}\right)^{\mathrm{d}}\end{array}$ & 0.47 & 4.2 & 0.77 & $\cdots$ & 4.9 \\
\hline $\begin{array}{l}\text { Column Density } \\
\left(\text { atoms } \mathrm{cm}^{-2}\right)^{\mathrm{d}}\end{array}$ & $1.10 \times 10^{19}$ & $6.83 \times 10^{19}$ & $7.45 \times 10^{19}$ & $\cdots$ & $6.67 \times 10^{19}$ \\
\hline $\begin{array}{l}\text { Peak Column Density } \\
\left(\text { atoms } \mathrm{cm}^{-2}\right)^{\mathrm{d}}\end{array}$ & $2.62 \times 10^{19}$ & $1.21 \times 10^{20}$ & & $\cdots$ & $1.70 \times 10^{20}$ \\
\hline $\operatorname{Mass}\left(\mathcal{M}_{\odot}\right)^{\mathrm{d}}$ & $1.6 \times 10^{8}$ & $14.0 \times 10^{8}$ & $2.5 \times 10^{8}$ & $11.5 \times 10^{8}$ & $16.3 \times 10^{8}$ \\
\hline Pressure $/ \mathrm{k}\left(\mathrm{K} \mathrm{cm}^{-3}\right)^{\mathrm{f}}$ & 5 & 100 & 50 & 91 & 39 \\
\hline
\end{tabular}

${ }^{\mathrm{a}}$ The central region of component B. See $\S 5.2 .1$ and Fig. 2 .

${ }^{\mathrm{b}}$ The uncertainty ( $\S .1$ ) is 10 to $15 \%$. With respect to Cloud $\mathrm{C}$ see the end of $\S 4.1 .1$.

${ }^{\mathrm{c}}$ At a distance of $37.6 \mathrm{Mpc}, 1^{\prime \prime}$ is equivalent to $182 \mathrm{pc}$.

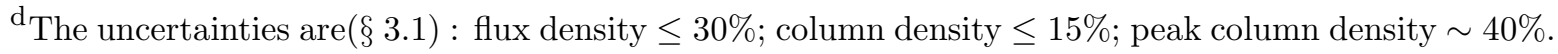
The uncertainty for mass is $\sim 33 \%$ ( $(4.1 .1)$.

${ }^{\mathrm{e}}$ For cloud $\mathrm{C}$ the column density value is the average of the north and south components which, separately, are $4.58 \times 10^{19}$ atoms $\mathrm{cm}^{-2}$ (north) and $8.76 \times 10^{19}$ atoms $\mathrm{cm}^{-2}$ (south).

${ }^{\mathrm{f}}$ The uncertainty is $43 \%$ ( $\left.\S 5.2 .1\right)$. Pressure has been divided by the Boltzmann Constant k. 
Table 4. Measured HI characteristics of the enhancements in the Vela Cloud. See Figure 5 for the relative positions of these enhancements. Measurements, analysis, and assessment of uncertainties are described in 93.2 .

\begin{tabular}{|c|c|c|c|c|c|}
\hline Enhancement & B1 & $\mathrm{B} 2$ & $\mathrm{C} 1 \mathrm{z}$ & C1y & $\mathrm{C} 2$ \\
\hline \multicolumn{6}{|l|}{$\operatorname{Position}(\mathrm{J} 2000)^{\mathrm{a}}$} \\
\hline$\alpha\left({ }^{\mathrm{hms}}\right)$ & 102827.2 & 102835.3 & 102813.2 & 102813.2 & 102827.8 \\
\hline$\delta\left({ }^{\circ \prime \prime \prime}\right)$ & -440817 & -440850 & -441728 & -441715 & -441613 \\
\hline $\begin{array}{l}\text { Central Velocity } \\
\left(\mathrm{km} \mathrm{s}^{-1}\right)\end{array}$ & $2906 \pm 3$ & $2908_{-9}^{+3}$ & $2807 \pm 7$ & $2835 \pm 7$ & $2815 \pm 3$ \\
\hline $\begin{array}{ll}\text { Peak } & \text { Intensity } \\
\text { r.m.s. } & \end{array}$ & 3 & 2 & 4 & 3 & 3 \\
\hline $\mathrm{FWHM}^{\mathrm{d}}\left(\mathrm{km} \mathrm{s}^{-1}\right)$ & $31 \pm 5$ & $28 \pm 2$ & $20 \pm 6$ & $21 \pm 6$ & $44 \pm 1$ \\
\hline $\begin{array}{l}\text { Integrated Flux } \\
\text { Density } \\
\left(\mathrm{Jy} \times \mathrm{km} \mathrm{s}^{-1}\right)\end{array}$ & 0.26 & 0.23 & 0.28 & 0.22 & 0.40 \\
\hline
\end{tabular}

aThe uncertainty in position is $26^{\prime \prime}(\S 3.2)$.

${ }^{b}$ These heliocentric radial velocities are the central velocities of single Gaussian profiles, except for C1. C1's two velocity peaks are labeled $\mathrm{z}$ and $\mathrm{y}$ and blend at the $50 \%$ level. Note, B2 is visually most delineated at $2897 \mathrm{~km} \mathrm{~s}^{-1}$.

${ }^{\mathrm{c}}$ This is the number of r.m.s. at which the peak intensity occurs above the mean intensity of the surrounding diffuse host component. See $\S 3.2$.

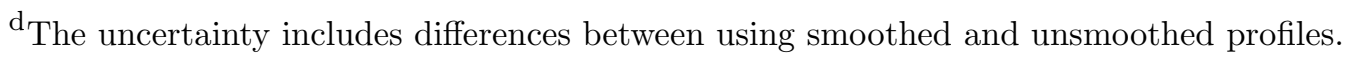

${ }^{\mathrm{e}}$ The uncertainty is $<0.06 \mathrm{Jy} \times \mathrm{km} \mathrm{s}^{-1} ; \S 3.2$. 
Table 5. Derived Parameters for Enhancements. The integrated flux density used to derive the mass associated with HI emission is listed in Table 4. The Bonnor-Ebert mass, $\mathcal{M}\left(\mathrm{r}_{\mathrm{c}}\right)$, associated with an isothermal sphere on the verge of collapse, is derived in $\S 5.3$.

\begin{tabular}{lccc}
\hline \hline & \multicolumn{3}{c}{ Enhancements } \\
Parameter & $\mathrm{B} 1$ & $\mathrm{~B} 2$ & $\mathrm{C} 2$ \\
& & & \\
\hline$\sigma_{o}{ }^{\mathrm{a}}\left(\mathrm{km} \mathrm{s}^{-1}\right)$ & 13.2 & 11.9 & 18.7 \\
$\mathcal{M}_{\mathrm{HI}}{ }^{\mathrm{b}}\left(\mathcal{M}_{\odot}\right)$ & $0.9 \times 10^{8}$ & $0.8 \times 10^{8}$ & $1.3 \times 10^{8}$ \\
$\mathcal{M}\left(\mathrm{r}_{\mathrm{c}}\right)^{\mathrm{c}}\left(\mathcal{M}_{\odot}\right)$ & $1.02 \times 10^{9}$ & $0.69 \times 10^{9}$ & $5.68 \times 10^{9}$ \\
$\frac{\mathcal{M}\left(\mathrm{r}_{\mathrm{c}}\right)}{\mathcal{M}_{\mathrm{HI}}}$ & 11 & 9 & 44 \\
\hline
\end{tabular}

aThe velocity dispersion in an enhancement $\sigma_{o}$ is derived using the FWHM listed in Table 4 .

${ }^{\mathrm{b}}$ The uncertainty is $\leq 29 \%$. The measurement is described in $\S 3.2$ and the uncertainty in $\S 4.2$.

${ }^{\mathrm{c}}$ The uncertainty is $\leq 43 \% ; \S 5.3$. 